\title{
The poset of graphs ordered by induced containment
}

\author{
Jason P. Smith ${ }^{1}$ \\ Department of Computer and Information Sciences, University of Strathclyde, \\ Glasgow, UK.
}

\begin{abstract}
We study the poset $\mathcal{G}$ of all unlabelled graphs with $H \leq G$ if $H$ occurs as an induced subgraph in $G$. We present some general results on the Möbius function of intervals of $\mathcal{G}$ and some results for specific classes of graphs. This includes a case where the Möbius function is given by the Catalan numbers, which we prove using discrete Morse theory, and another case where it equals the Fibonacci numbers, therefore showing that the Möbius function is unbounded. A classification of the disconnected intervals of $\mathcal{G}$ is presented, which gives a large class of non-shellable intervals. We also present several conjectures on the structure of $\mathcal{G}$.
\end{abstract}

\section{Introduction}

Given any set of combinatorial objects and a suitable notion of containment of one such object in another we can define a poset. Many such posets have been studied in the literature, such as the posets of words with subword order [Bjö90], the permutation pattern poset [BJJS11, Smi17], the poset of graphs with minor order [RS04], and many more. In this paper we introduce and study the poset $\mathcal{G}$ of all graphs ordered by induced containment, that is, $\mathcal{G}$ contains all unlabelled finite graphs and $H \leq G$ if $H$ is an induced subgraph of $G$. Where an induced subgraph of $G$ is given by a subset $S$ of the vertices of $G$ and all of the edges of $G$ connecting pairs of vertices in $S$. We say that $G$ contains $H$ if $H \leq G$ and we consider two subgraphs to be the same if they are isomorphic. We refer the reader to $[\mathrm{BM} 76]$ for further background in graph theory and any terms not defined here.

We study the Möbius function and topology of $\mathcal{G}$, and present some results and conjectures. The poset $\mathcal{G}$ has a countably infinite number of elements and is locally finite, so we focus our attention on the intervals $[a, b]=$ $\{z \in \mathcal{G} \mid a \leq z \leq b\}$, see Figure 2.1. We also consider the poset $\mathcal{G}^{c}$ of all connected graphs, which is an induced subposet of $\mathcal{G}$, and we denote intervals of $\mathcal{G}^{c}$

Email address: jason.smith@abdn.ac.uk (Jason P. Smith)

${ }^{1}$ This research was supported by the EPSRC Grant EP/M027147/1. 
by $[a, b]^{c}$. Unless otherwise specified we allow graphs to have loops and multiple edges.

Whilst the topology of the poset $\mathcal{G}$ does not seem to have been studied, the poset has been considered from a model theoretic perspective in [Wir16]. There are also many other posets of graphs that have been investigated. In [Tha06] the poset of all induced connected subgraphs of a graph $G$ is considered in relation to the graph reconstruction conjecture. This is equivalent to the interval $\left[K_{1}, G\right]^{c}$ in $\mathcal{G}^{c}$, where $K_{1}$ is the graph with a single vertex, but the topology and Möbius function of these posets was not considered. In [KS96] the poset $C(G)$ is defined on a graph $G$ with the same partial order considered here, but graphs are not considered the same if they are isomorphic. Such a poset has a simpler structure than $\mathcal{G}$ because subgraphs occur exactly once in the parent graph. In [ST17] the posets of connected labelled graphs on $n$ vertices ordered by non-induced containment is shown to be Sperner. Another poset that has been considered previously is that of graphs ordered by the minor relation, that is, $G \leq H$ if $G$ is a graphical minor of $H$. This is well known to be a partial order and was famously shown to be a well-quasi ordering by the Robertson-Seymour Theorem [RS04].

A finite poset $P$ is shellable if there is a "nice" ordering of the maximal chains, that is, the maximal totally ordered subsets of $P$, see [Wac07] for a formal definition of shellability. A poset is not shellable if it contains any disconnected subintervals of rank at least 3 . We give a classification of the disconnected intervals of $\mathcal{G}$, which is similar to that given for permutation patterns in [MS15]. Moreover, we present a large class of non-shellable intervals of $\mathcal{G}$, but we conjecture that the proportion of intervals that are shellable tends to 1 as the rank of the intervals increases.

Note that $\mathcal{G}$ is a ranked poset, that is, all maximal chains of each interval have the same length, and is not a lattice. It is straightforward to see that the rank function is simply the order $|G|$ of a graph $G$, that is, the number of vertices. Moreover, it can easily be seen in Figure 2.1 that $\mathcal{G}$ is not a lattice, as there are multiple pairs which do not have a unique join.

In Section 2 we give a classification of the disconnected intervals $[H, G]$ of $\mathcal{G}$ based on the set of occurrences of $H$ in $G$. In Section 3 we give some general results on the Möbius function of intervals of $\mathcal{G}$. In Section 3.1 we consider intervals of $\mathcal{G}$ and $\mathcal{G}^{c}$ between some well known graphs, such as the complete graphs $K_{n}$, the cycle graphs $C_{n}$ and the empty graphs $\bar{K}_{n}$, that is, the graphs with no edges. In Section 4 we consider intervals between graphs of disjoint paths. We prove a result where the Möbius function is given by the Catalan numbers, using discrete Morse theory, and another case where it is given by the Fibonacci numbers. As a corollary we get that the Möbius function is unbounded on $\mathcal{G}$. In Section 5 we finish with some conjectures about $\mathcal{G}$.

\section{Disconnected Intervals}

In this section we consider the disconnected intervals of $\mathcal{G}$ and $\mathcal{G}^{c}$. An interval $[x, y]$ is disconnected if the interior $(x, y):=[x, y] \backslash\{x, y\}$ can be split into 
two non-empty sets $A$ and $B$, which we call components, with $a \not \leq b$ and $b \not \leq a$ for all $a \in A$ and $b \in B$. A disconnected subinterval is non-trivial if it has a rank of at least 3, and it is shown in [Bjö80, Proposition 4.2] that a non-trivial disconnected poset is not shellable.

In [MS15] a classification of the disconnected intervals of the permutation poset is given based on splitting the set of occurrences into two disjoint sets, and this result is generalised in [Smi16b] to general pattern posets. In this section we introduce and apply an analogous result to the poset of graphs. First we need to define an occurrence in this setting.

Given a graph $G$ we denote the set of vertices and set of edges of $G$ by $V(G)$ and $E(G)$, respectively. We arbitrarily assign the labels $[|G|]:=\{1, \ldots,|G|\}$ to the elements of $V(G)$. This labelling is just to record which vertices give an induced subgraph $H$, but we do not consider the graph as a labelled graph. Given a set $\eta \subseteq[|G|]$ we denote the subgraph of $G$ induced by $\eta$ as $G[\eta]$ and say that $\eta$ is an occurrence of $H$ in $G$ if $G[\eta] \cong H$. Let $O_{H, G}$ be the set of all occurrences of $H$ in $G$. Given any $\eta \in O_{H, G}$ define the set $Z(\eta):=[|G|] \backslash \eta$ and for any $A \subseteq O_{H, G}$ let $Z(A)=\bigcup_{\eta \in A} Z(\eta)$. For ease of notation we will often denote an occurrence $\{a, b, c\}$ as simply $a b c$. Next we introduce the notion of zero-splitness, first introduced in [MS15, Proposition 5.3], although not with the same name.

Definition 2.1. The interval $[H, G]$ is zero-split if $O_{H, G}$ can be split into two non-empty disjoint sets $A$ and $B$ such that $Z(A) \cap Z(B)=\emptyset$. We call $A$ and $B$ a zero-split partition of $[H, G]$.

The interval $[H, G]$ is strongly zero-split if there is a zero-split partition $A$ and $B$ and there does not exist $\eta \in A, \phi \in B$ and $i, j \in[|G|]$ such that $G[\eta \cup i] \cong G[\phi \cup j]$.

Example 2.2. Consider the interval $\left[C_{4}, G\right]$ where

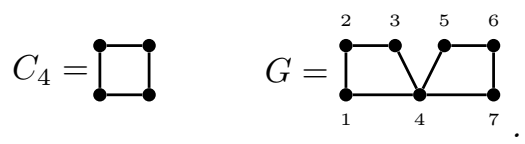

So $O_{C_{4}, G}=\{1234,4567\}$ and there is a zero-split partition $A=\{1234\}$ and $B=\{4567\}$. However, the interval is not strongly zero-split because

$$
G[1234 \cup 7] \cong G[4567 \cup 1] \cong ? \text {. }
$$

We can now present the main theorem of this section.

Theorem 2.3. An interval $[H, G]$, with $|G|-|H|>2$, is disconnected if and only if it is strongly zero-split.

Proof. Suppose $[H, G]$ is strongly zero-split with partition $E_{1}$ and $E_{2}$. Define the sets $P_{1}$ and $P_{2}$ such that $X \in P_{i}$ if there is an $\alpha \in O_{X, G}$ with $Z(\alpha) \subset Z(\eta)$ for some $\eta \in E_{i}$. Every graph in $(H, G)$ can be obtained by deleting some subset of $Z(\eta)$ for some $\eta \in O_{H, G}$, so $(H, G)=P_{1} \cup P_{2}$. 


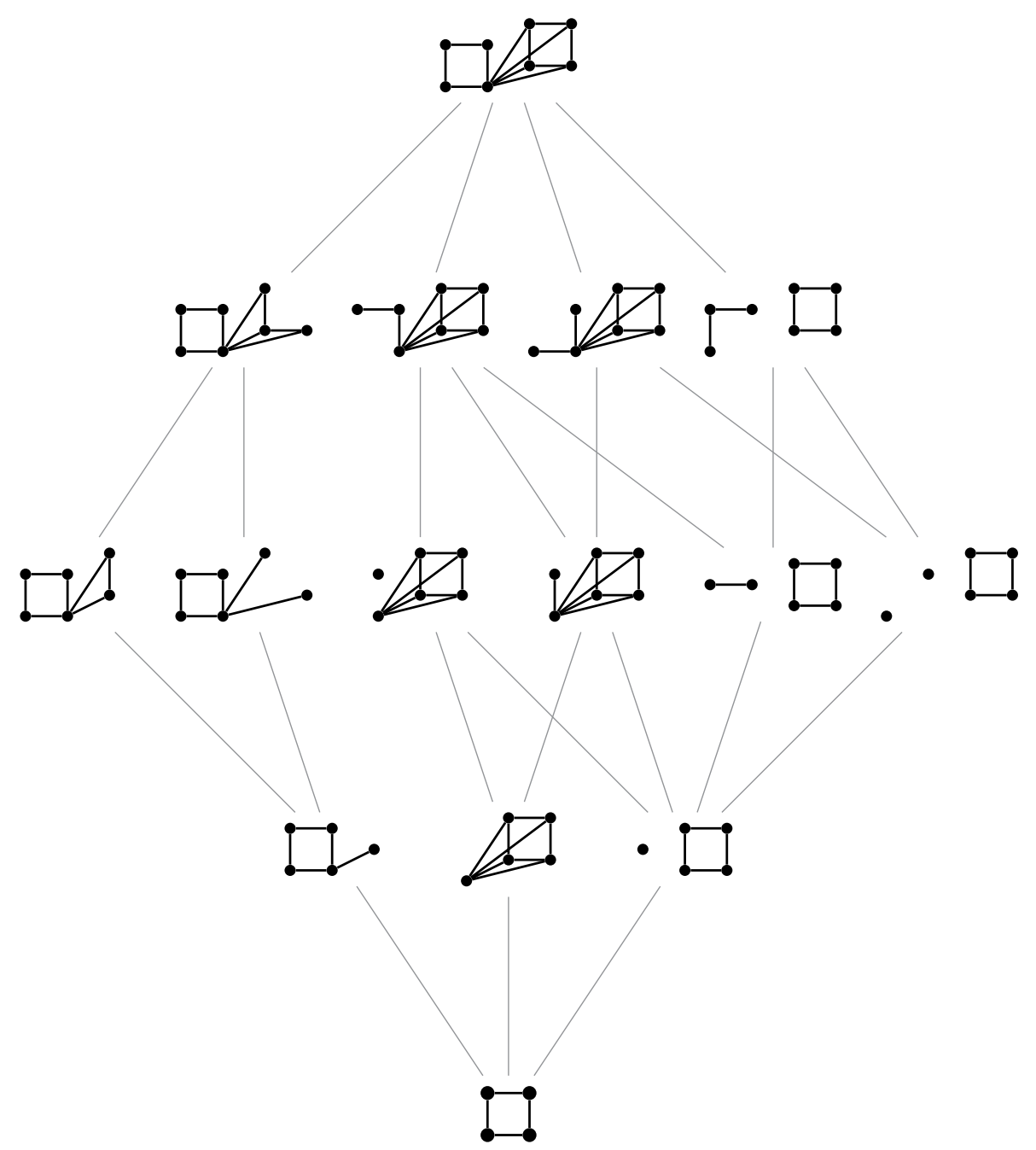

Figure 2.1: The disconnected interval $\left[C_{4}, H\right]$ in $\mathcal{G}$, for any $v \in V\left(C_{4}\right)$, where $H=D_{v}\left(C_{4}\right)$ as defined in Definition 2.6 . 
We claim that $P_{1}$ and $P_{2}$ are disconnected components of $(H, G)$. First note that if $X \in P_{i}$ and $Y \leq X$, then $Y \in P_{i}$. To see this consider $\alpha \in O_{X, G}$ with $Z(\alpha) \subset Z(\eta)$ for some $\eta \in E_{i}$. There is a $\beta \in O_{Y, G}$ with $Z(\beta) \supseteq Z(\alpha)$. Moreover, $Z(\beta) \subset Z(\zeta)$ for some $\zeta \in O_{H, G}$ which implies $Z(\zeta) \cap Z(\eta) \supseteq Z(\alpha)$. As $Z(\eta)$ and $Z(\zeta)$ have non-empty intersection, $\eta$ and $\zeta$ cannot be in separate sets of the partition, which implies $\zeta \in E_{i}$ thus $Y \in P_{i}$.

For a contradiction suppose $P_{1}$ and $P_{2}$ are not disconnected so there exists a comparable pair $p_{1} \in P_{1}$ and $p_{2} \in P_{2}$, and without loss of generality suppose $p_{1} \leq p_{2}$. This implies that $p_{1} \in P_{1} \cap P_{2}$, and consider any $A \leq p_{1}$ with $|A|=|H|+1$, then $A \in P_{1} \cap P_{2}$. However, this violates the strongly zerosplit condition as it implies there is a pair $e_{1} \in E_{1}$ and $e_{2} \in E_{2}$ both of which are contained in occurrences of $A$ in $G$. Therefore, $P_{1}$ and $P_{2}$ are disconnected components.

Suppose that $(H, G)$ is disconnected with components $P_{1}$ and $P_{2}$, and let

$$
E_{i}=\left\{\eta \in O_{H, G} \mid Z(\eta) \subseteq \bigcup_{B \in P_{i}} Z\left(O_{B, G}\right)\right\} .
$$

So $E_{i}$ is the set of occurrences $\eta$ from which an element of $P_{i}$ can be obtained by removing a subset of $Z(\eta)$ from $G$. The sets $E_{1}$ and $E_{2}$ form a zero-split partition because if $i \in Z\left(E_{1}\right) \cap Z\left(E_{2}\right)$ then $G-i$ is in both $P_{1}$ and $P_{2}$, which gives a contradiction. To see this is a strongly zero-split partition suppose there is a graph $X$ that can be obtained by adding a vertex to occurrences from different sets $E_{1}$ and $E_{2}$, this would imply that $X \in P_{1} \cap P_{2}$, again giving a contradiction. Therefore, $[H, G]$ is strongly zero-split.

Corollary 2.4. If $|G|>2|H|$ then $[H, G]$ is connected.

Proof. For every pair $\eta, \zeta \in O_{H, G}$ we have $|Z(\eta) \cap Z(\zeta)|>0$ so $[H, G]$ is not zero-split and the result follows by Theorem 2.3.

We get the following corollary by applying an analogous proof to that used in Theorem 2.3:

Corollary 2.5. Consider an interval $[H, G]^{c}$ in $\mathcal{G}^{c}$ with $|G|-|H|>2$, then $[H, G]^{c}$ is disconnected if and only if it is strongly zero-split.

We can use Theorem 2.3 to construct an infinite class of disconnected intervals, using the following construction. See Figure 2.1 for an example of $D_{v}(H)$.

Definition 2.6. Given a graph $H$ and vertex $v \in V(H)$ define the graph $D_{v}(H)$ with vertex and edge sets:

$$
\begin{gathered}
V\left(D_{v}(H)\right)=\{1,2\} \times V(H), \\
E\left(D_{v}(H)\right)=\{((i, x),(i, y)) \mid(x, y) \in E(H), i \in\{1,2\}\} \\
\cup\{((1, v),(2, x)) \mid x \in V(H)\},
\end{gathered}
$$

that is, $D_{v}(H)$ is constructed by taking two copies of $H$ and connecting all vertices of one copy to the vertex $v$ in the other copy. 
Define a pendant in a graph as a vertex with exactly one neighbour.

Lemma 2.7. Consider a connected graph $H$, with $|H| \geq 3$, that does not contain $C_{3}$ and has no pendants. The interval $\left[H, D_{v}(H)\right]$ is disconnected, for all $v \in V(H)$.

Proof. There are clearly at least two occurrences of $H$ in $D_{v}(H)$, namely the two copies of $H$ that $D_{v}(H)$ is constructed from. Let $A$ be the occurrence of $H$ containing $v$, and $B$ be the other occurrence. Suppose there is a third occurrence $C$, then $C$ must contain vertices of both $A$ and $B$, and must contain $v$, since otherwise it is disconnected. If there are no edges between elements of $C \cap B$, then each of these vertices has a single neighbour in $C$ which is $v$, thus they are pendants in $H$ which is not allowed. If there are edges between two elements $x, y \in C \cap B$, then $x, y, v$ is an occurrence of $C_{3}$ in $C$, and thus in $H$ which is not allowed. Therefore, there is no third occurrence of $H$.

By the definition of $D_{v}(H)$ the occurrences $A$ and $B$ are zero-split. Moreover, in $A \cup i$ the vertex $i$ is a pendant for any $i \in D_{v}(H) \backslash A$, in $B \cup j$ the vertex $j$ has $|H|$ neighbours if $j=v$ and no neighbours for any other $j \in D_{v}(H) \backslash B$. Therefore $A \cup i \neq B \cup j$ for any $i$ and $j$, so $\left[H, D_{v}(H)\right]$ is strongly zero-split, and thus disconnected by Theorem 2.3 .

See Figure 2.1 for an example of Lemma 2.7. A poset is non-shellable if it contains a non-trivial disconnected subinterval, that is, a subinterval of rank at least 3 which is disconnected. Therefore, we can use Lemma 2.7 to get an infinite class of non-shellable intervals.

Corollary 2.8. Consider a connected graph $H$, with $|H| \geq 3$, that does not contain $C_{3}$ and has no pendants. As $n$ tends to infinity the probability that $[H, G]$,

where $|G|=n$, contains a non-trivial disconnected subinterval, and thus is not shellable, tends to 1.

Proof. For any fixed graph $H$ the probability that $H$ occurs as an induced subgraph in a graph $G$ tends to 1 as $|G|$ tends to infinity, see [Die12, Section 11, Exercise 12]. Therefore, the probability that $G$ contains $D_{v}(H)$ tends to 1 as $|G|$ tends to infinity, which implies $[H, G]$ contains the subinterval $\left[H, D_{v}(H)\right]$, which is disconnected by Lemma 2.7 .

Whilst the class of connected graphs not containing $C_{3}$ and with no pendants is infinite, it is also a vanishingly small proportion of all graphs. Which implies that the proportion of intervals of $\mathcal{G}$ satisfying the conditions of Corollary 2.8 is vanishingly small. So the result does not provide much information about whether or not most intervals of $\mathcal{G}$ are shellable.

\section{Möbius Function}

In this section we give some results on the Möbius function of intervals of $\mathcal{G}$. Define the half-open interval $[a, b):=[a, b] \backslash\{b\}$. The Möbius function of a 
poset $P$ is defined recursively by $\mu_{P}(a, a)=1$ for all $a, \mu_{P}(a, b)=0$ if $a \not \leq b$ and if $a<b$ then

$$
\mu_{P}(a, b)=-\sum_{c \in[a, b)} \mu_{P}(a, c) .
$$

The dual $P^{*}$ of a poset $P$ is the poset with the same elements of $P$ and the partial order is reversed, that is, $a \leq_{P^{*}} b$ if and only if $a \geq_{P} b$. It is well known that $\mu_{P^{*}}(b, a)=\mu_{P}(a, b)$. We use $\mu$ to denote $\mu_{\mathcal{G}}$ and $\mu_{c}$ to denote $\mu_{\mathcal{G}^{c}}$, and $\mu^{*}$ and $\mu_{c}^{*}$ to denote Möbius function on their duals. See Figure 3.1 for an example of the Möbius function on $\mathcal{G}$.

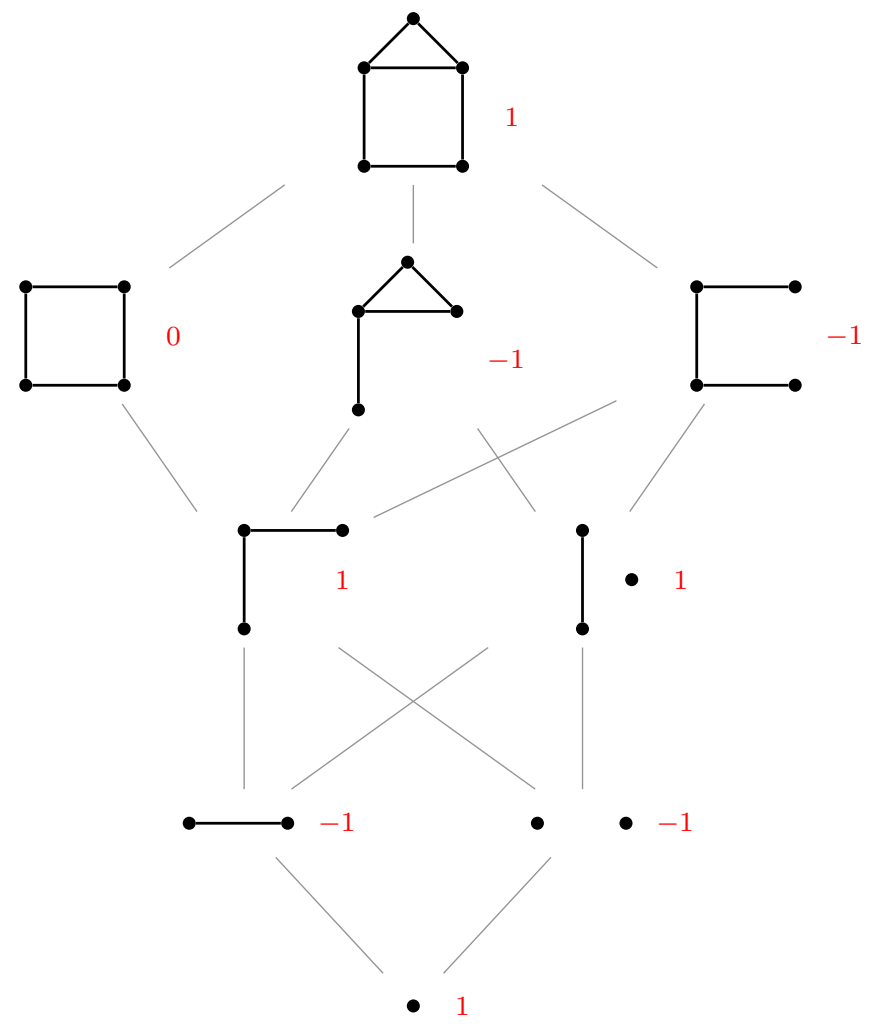

Figure 3.1: The interval $\left[K_{1}, H\right]$ in $\mathcal{G}$, where $H$ is the house graph, with $\mu\left(K_{1}, X\right)$ in red.

First we look at a condition on $G$ which results in $\mu(H, G)=0$ for all $|H|<|G|-1$. An automorphism on a graph $G$ is a map $f$ from $V(G)$ onto itself such that $(u, v)$ is an edge if and only if $(f(u), f(v))$ is an edge. A graph is locally finite if every vertex has a a finite number of neighbours.

Definition 3.1. A graph is vertex transitive if for every pair of vertices $v_{1}$ and $v_{2}$ there is an automorphism that maps $v_{1}$ to $v_{2}$. 
The coatoms of an interval $[H, G]$ are the maximal elements of $(H, G)$. The following theorem allows us to consider what the coatoms of $[H, G]$ are when $G$ is vertex transitive.

Theorem 3.2. [Tho87] Let $G$ be a locally finite graph without isolated vertices. Then $G$ is vertex transitive if and only if deleting any vertex gives the same graph up to isomorphism.

If we restrict to only finite graphs then we can remove the condition on isolated vertices, as we now show.

Corollary 3.3. Let $G$ be a finite graph, then $G$ is vertex transitive if and only if deleting any vertex gives the same graph up to isomorphism.

Proof. If $G$ is finite and has no isolated vertices, then the result follows by Theorem 3.2. If $G$ is finite and vertex transitive, then it must be regular, that is, all vertices have the same degree. So if there is an isolated vertex all vertices must be isolated, so $G$ is the graph with no edges, which implies all vertex deleted subgraphs are isomorphic. The converse follows by a similar argument.

Corollary 3.3 implies that if $G$ is a vertex transitive graph then it contains, up to isomorphism, exactly one graph of order one less. Moreover, it is well known that if an interval $[x, y]$ has a single coatom, and at least 3 elements, then $\mu(x, y)=0$, which immediately implies the following result.

Proposition 3.4. If a graph $G$ is vertex transitive, then $\mu(H, G)=0$ for any $|H|<|G|-1$.

A graph is simple if it has no loops nor multiple edges. The complement $\bar{G}$ of a simple graph $G$ has the same vertex set as $G$ and $(a, b)$ is an edge in $\bar{G}$ if and only if it is not an edge in $G$. Applying the complement operation to an interval of simple graphs does not change the Möbius function.

Lemma 3.5. If $H$ and $G$ are simple graphs, then $[H, G]$ is isomorphic to $[\bar{H}, \bar{G}]$, so $\mu(H, G)=\mu(\bar{H}, \bar{G})$.

Proof. It is trivial to see that $g_{1}, \ldots, g_{|H|}$ is an occurrence of $H$ in $G$ if and only if it is an occurrence of $\bar{H}$ in $\bar{G}$. Therefore, $H \leq G$ if and only if $\bar{H} \leq \bar{G}$, which implies the result.

\subsection{Well known graphs}

In this subsection we consider the Möbius function of intervals of some well known graphs. First note that the complete, empty and cycle graphs are vertex transitive, so by Proposition 3.4 we get:

Lemma 3.6. Consider any $n>0$ and graph $H$ with $|H| \notin\{n-1, n\}$, then:

$$
\mu\left(H, K_{n}\right)=\mu\left(H, \overline{K_{n}}\right)=\mu\left(H, C_{n}\right)=0 .
$$


Next we consider the intervals $[\emptyset, G]$, where $\emptyset$ is the null graph, that is, the graph with no vertices.

Lemma 3.7. If a graph $G$ has no loops, then:

$$
\mu(\emptyset, G)= \begin{cases}1, & \text { if }|G|=0 \\ -1, & \text { if }|G|=1 \\ 0, & \text { if }|G|>1\end{cases}
$$

Proof. The cases $|G| \leq 1$ follow trivially. Suppose $|G|>1$, then the interval $(\emptyset, G)$ has a unique minimal element $K_{1}$, which implies $\mu(\emptyset, G)=0$.

If $G$ contains loops then Lemma 3.7 does not hold as there are multiple minimal elements in $(\emptyset, G)$, which are the graphs with a single vertex and $x$ loops, for some $x \geq 0$.

Next we consider bipartite graphs.

Lemma 3.8. If $G$ is a non-empty bipartite graph with $|G|>2$, then

$$
\mu\left(K_{1}, G\right)=-\mu\left(K_{2}, G\right) .
$$

Proof. Note that $K_{2}$ and $\overline{K_{2}}$ are contained in $G$ and in every element of $\left(K_{2}, G\right)$. The only elements in $\left(K_{1}, G\right) \backslash\left(K_{2}, G\right)$ are $K_{2}, \overline{K_{2}}$ and possibly some larger empty graphs $\overline{K_{a}}$. Note that $\mu\left(K_{1}, \overline{K_{a}}\right)=0$, for any $a>2$, by Lemma 3.6. Let $Q$ be the poset obtained by removing all $\overline{K_{a}}$, with $a>2$, from $\left(K_{1}, G\right)$, then $\mu_{Q}\left(K_{1}, G\right)=\mu\left(K_{1}, G\right)$. The poset $Q$ consists of $\left(K_{2}, G\right) \cup\left\{K_{2}, \overline{K_{2}}\right\}$, and every element of $\left(K_{2}, G\right)$ contains both $K_{2}$ and $\overline{K_{2}}$. We consider the dual poset $Q^{*}$ and let $\mathcal{I}=\left[G, \overline{K_{2}}\right] \cup K_{2}$, then

$$
\mu\left(K_{1}, G\right)=\mu_{Q^{*}}\left(G, K_{1}\right)=-\sum_{X \in \mathcal{I}} \mu_{Q^{*}}(G, X)=-\mu_{Q^{*}}\left(G, K_{2}\right)=-\mu\left(K_{2}, G\right) .
$$

Remark 3.9. We can also prove Lemma 3.8 using a topological argument similar to that used in [Smi16a, Theorem 4.2] to prove a result on the permutation pattern poset. To do so we show that $\left(K_{1}, G\right)$ is homotopy equivalent to $Q$ by the Quillen Fiber lemma, see [Koz08, Theorem 15.28], and $Q$ is a suspension of $\left(K_{2}, G\right)$, which implies the result.

Let $K_{a, b}$ be the complete bipartite graph with parts of size $a$ and $b$.

Lemma 3.10.

$$
\mu\left(K_{a_{1}, a_{2}}, K_{b_{1}, b_{2}}\right)= \begin{cases}1, & \text { if } a_{1}=b_{1} \& a_{2}=b_{2} \\ -1, & \text { if }\left(b_{1}-a_{1}\right)+\left(b_{2}-a_{2}\right)=1 \& b_{1} \geq a_{1} \& b_{2} \geq a_{2} \\ 1, & \text { if } b_{1}-a_{1}=b_{2}-a_{2}=1 \& a_{1} \neq a_{2} \\ 0, & \text { otherwise }\end{cases}
$$


Proof. The first two cases follow trivially as they are rank 0 and 1 intervals. If $b_{1}-a_{1}=b_{2}-a_{2}=1$ the interval has rank 2 and $\left(K_{a_{1}, a_{2}}, K_{b_{1}, b_{2}}\right)$ contains $K_{a_{1}+1, a_{2}}$ and $K_{a_{1}, a_{2}+1}$, but if $a_{1}=a_{2}$ then these are isomorphic, this implies the result for rank 2 intervals.

Suppose the interval has rank $r>2$. If $a_{1}=a_{2}$ or $b_{1}=b_{2}$, then in the open interval there is a unique minimal element $K_{a_{1}+1, a_{2}}$ or maximal element $K_{b_{1}-1, b_{2}}$, respectively, which implies the result. Otherwise, by induction we can see that the only elements in $\left[K_{a_{1}, a_{2}}, K_{b_{1}, b_{2}}\right)$ with non-zero Möbius function are $K_{a_{1}, a_{2}}, K_{a_{1}+1, a_{2}}, K_{a_{1}, a_{2}+1}$ and $K_{a_{1}+1, a_{2}+1}$. Therefore, $\mu\left(K_{a_{1}, a_{2}}, K_{b_{1}, b_{2}}\right)=$ $1-1-1+1=0$.

We can combine Lemma 3.10 with Lemma 3.8 to get the following corollary.

Corollary 3.11. For any $a, b$ with $a+b>3$ :

$$
\mu\left(K_{1}, K_{a, b}\right)=0
$$

We can also consider the complete multipartite graphs $K_{b_{1}^{i_{1}}, \ldots, b_{k}^{i_{k}}}$ with $i_{j}$ parts of size $b_{j}$, for all $1 \leq j \leq t$.

Lemma 3.12. Given any integers $a, n, t>0$ we have $\mu\left(H, K_{t^{n}}\right)=0$, for all $|H|<$ tn -1 , and $\mu\left(K_{1^{a+n}}, K_{1^{a}, t^{n}}\right)=0$.

Proof. The graph $K_{t^{n}}$ is vertex transitive, which gives the first part of the statement. The only vertices that can be deleted from $K_{1^{a}, t^{n}}$ are those in the parts of size $t$, and deleting any vertex from any of those parts gives the same graph up to isomorphism. So there is a unique maximal element in the open interval.

\section{Posets between graphs of disjoint paths}

In this section we consider graphs which are a collection of disjoint paths, or equivalently acyclic graphs where every vertex has degree at most 2 . Let $P_{a}$ denote the path graph of order $a$. We show that the Möbius function from $\overline{K_{n}}$ to $n$ disjoint copies of $P_{5}$ is equal to the $n$ 'th Catalan number $\mathcal{C}_{n}$. To show this we use discrete Morse theory, which we introduce in Section 4.2. We also show that the Möbius function from $\overline{K_{2}}$ to two disjoint copies of $P_{n}$ is equal to the $n$ 'th Fibonacci number $\mathcal{F}_{n}$.

Given a multiset $S$ of positive integers we define $S_{p}$ to be the graph $\sqcup_{i \in S} P_{i}$, where $\sqcup$ denotes the disjoint union. For ease of notation we often write $S_{p}$ as $\alpha_{p}$, where $\alpha$ is the word consisting of the letters of $S$ in decreasing order. To avoid confusion we use upper and lower case letters to denote the multisets and words, respectively. We also use $x_{p}^{n}$ to represent the graph made of $n$ disjoint copies of $P_{x}$, and $x^{n} y^{m}{ }_{p}=x_{p}^{n} \sqcup y_{p}^{m}$. For example, the graph $\{4,4\}_{p}$, also written $44_{p}$ or $4_{p}^{2}$, is two disjoint paths of length 4 , see Figure 4.1 for the interval $\left[11_{p}, 44_{p}\right]$. Let $\mathcal{P}$ be the subposet of $\mathcal{G}$ consisting of all graphs of disjoint paths, and let $\mathcal{P}_{\leq i}$ be the subposet of $\mathcal{P}$ of graphs where all paths have max length $i$. 


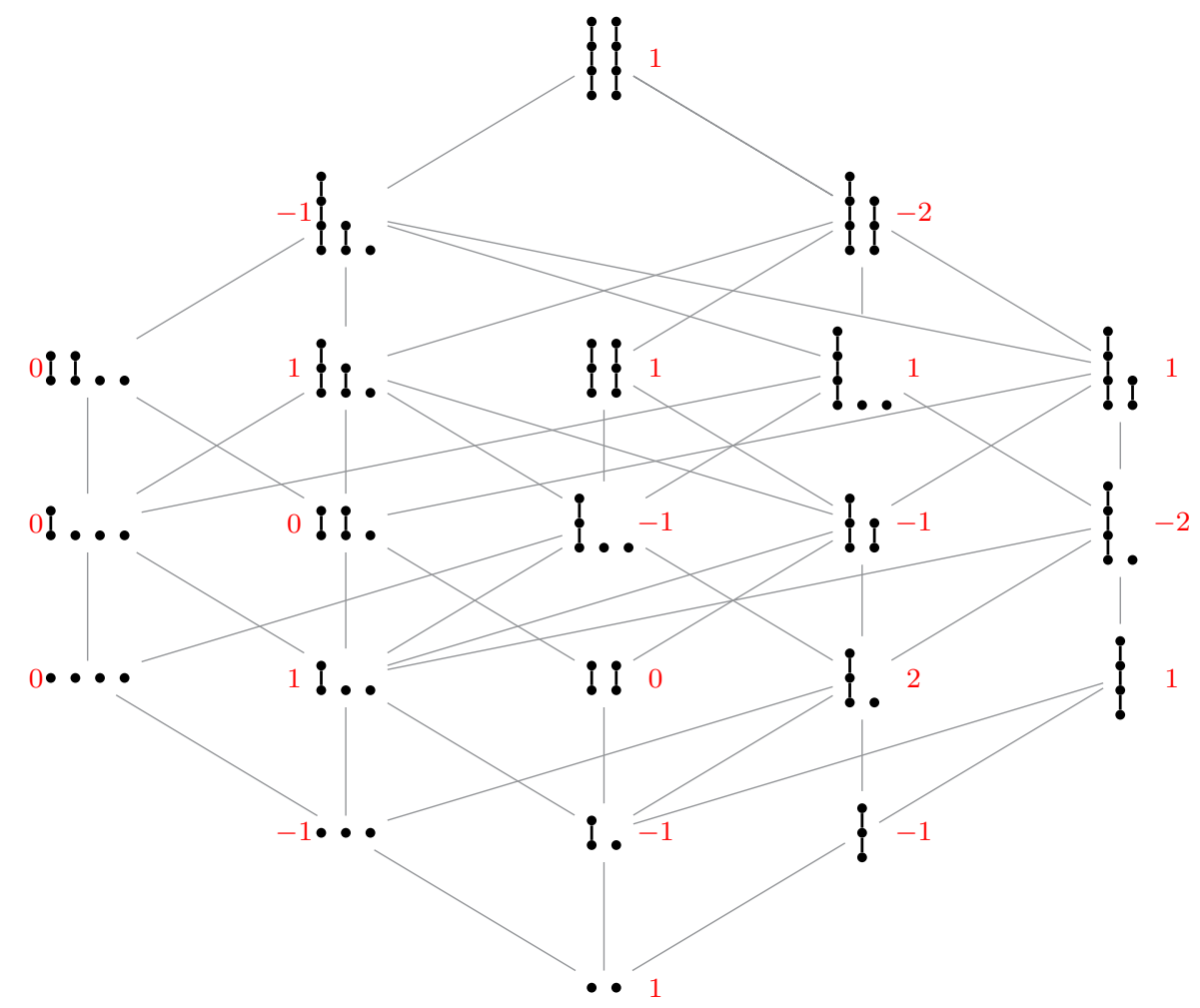

Figure 4.1: The interval $\left[11_{p}, 44_{p}\right]$, with $\mu\left(11_{p}, X\right)$ in red. 


\begin{tabular}{c|ccccccccc}
$x$ & 1 & 2 & 3 & 4 & 5 & 6 & 7 & 8 & 9 \\
\hline 1 & 1 & -1 & 1 & -1 & 1 & -1 & 1 & -1 & 1 \\
2 & 1 & 0 & 1 & 1 & 2 & 3 & 5 & 8 & \\
3 & 1 & 0 & 1 & -1 & 5 & -14 & 47 & & \\
4 & 1 & 0 & 1 & 1 & 14 & 81 & & & \\
5 & 1 & 0 & 1 & -1 & 42 & & & & \\
6 & 1 & 0 & 1 & 1 & & & & & \\
7 & 1 & 0 & 1 & & & & & & \\
8 & 1 & 0 & & & & & & & \\
9 & 1 & & & & & & & &
\end{tabular}

Table 1: The values of $\mu\left(1_{p}^{n}, x_{p}^{n}\right)$, for $n+x \leq 10$.

In Table 1 we display the Möbius function $\mu\left(1_{p}^{n}, x_{p}^{n}\right)$, for $x+n \leq 10$. We can see that for $n=2$ we get the Fibonacci numbers $\mathcal{F}_{x-2}$ and for $x=5$ we get the Catalan numbers $\mathcal{C}_{n}$. In this section we prove the following result which deals with the first five columns and first two rows of Table 1.

Theorem 4.1. Let $\mathcal{C}_{i}$ and $\mathcal{F}_{i}$ be the $i$ 'th Catalan and Fibonacci numbers, respectively. We can compute $\mu\left(1_{p}^{n}, x_{p}^{n}\right)$ for the following cases:

$$
\mu\left(1_{p}^{n}, x_{p}^{n}\right)= \begin{cases}(-1)^{x+n}, & \text { if } n=1 \text { or } x=4 \\ 1, & \text { if } x=1 \text { or } x=3 \\ 0, & \text { if } x=2 \quad \text { \& } n>1, \\ F_{x-2}, & \text { if } n=2, \\ \mathcal{C}_{n}, & \text { if } x=5 .\end{cases}
$$

An immediate corollary of Theorem 4.1 is:

Corollary 4.2. The Möbius function is unbounded on $\mathcal{G}$.

In the following two subsections we prove Theorem 4.1 by Corollary 4.4, Propositions 4.8, 4.14 and 4.16, and Lemma 4.17. In Section 4.1 we apply an inductive argument and in Section 4.2 we use discrete Morse theory.

\subsection{The cases $n=1$ and $n=2$}

We begin with the case $n=1$, that is, the interval $\left[1_{p}, x_{p}\right]$ from the singleton graph to the path graph of order $x$. To prove this case we prove a more general result on intervals $\left[m_{p}, x_{p}\right]$ between any two path graphs, from which the result for $n=1$ immediately follows by setting $m=1$.

Lemma 4.3. For any $0<m \leq x$ we have $\mu\left(m_{p}, x_{p}\right)=(-1)^{x-m}$.

Proof. We consider the dual poset and prove $\mu^{*}\left(x_{p}, m_{p}\right)=(-1)^{x-m}$. Fixing $x$, we proceed by induction decreasing the value of $m$. Clearly $\mu^{*}\left(x_{p}, x_{p}\right)=1$ 
and assume $\mu^{*}\left(x_{p}, \ell_{p}\right)=(-1)^{x-m-1}$, for $\ell=m+1$. In the dual poset the graph $m_{p}$ covers exactly two graphs $\ell_{p}$ and $m 1_{p}$, which is the graph obtained by adding an isolated vertex to $m_{p}$. It is straightforward to see $Y \leq^{*} m 1_{p}$ for every $Y \in\left(x_{p}, m_{p}\right)^{*} \backslash \ell_{p}$. So:

$$
\begin{aligned}
\mu^{*}\left(x_{p}, m_{p}\right)=-\left(\sum_{Y \in\left[x_{p}, m 1_{p}\right]^{*}} \mu^{*}\left(x_{p}, Y\right)+\mu^{*}\left(x_{p}, \ell_{p}\right)\right) & =-\mu^{*}\left(x_{p}, \ell_{p}\right) \\
& =-(-1)^{x-m-1}
\end{aligned}
$$

Corollary 4.4. For any $x \geq 1$ we have $\mu\left(1_{p}, x_{p}\right)=(-1)^{x-1}$.

Next we prove the Fibonacci case $n=2$. For ease of notation we denote $\mu\left(11_{p}, X\right)$ by $\mu^{11}(X)$. First we need some lemmas. In the proofs of the next four results we apply a similar argument each time, which we outline here. Consider an interval $I=[x, y]$, with $x \neq y$, and an element $Z \in(x, y)$, then by the definition of the Möbius function we know that $\sum_{w \in I} \mu(x, w)=0$, and when computing $\mu(x, y)$ we can ignore any $v \in I$ with $\mu(x, v)=0$. So for a given $I$ and $Z$ define the set:

$$
L_{I}^{Z}:=\{v \in[x, y) \mid v \not \subset Z \& \mu(x, v) \neq 0\},
$$

which gives us the formula

$$
\mu(x, y)=-\sum_{v \in L_{I}^{Z}} \mu(x, v) .
$$

So our approach is to find an appropriate $Z$, compute the elements of $L_{I}^{Z}$, and look at all the remaining elements $v \notin L_{I}^{Z}$, ignoring any for which $\mu(x, v)=0$.

Lemma 4.5. If $A$ is a multiset with $|A|=3$ and $1 \notin A$ or $|A|>3$, then $\mu^{11}\left(A_{p}\right)=0$.

Proof. The minimal cases for $A$ are $\{1,1,1,1\}$ or $\{2,2,2\}$. It is easy to see that $\mu^{11}\left(1111_{p}\right)=\mu^{11}\left(222_{p}\right)=0$. Assume the claim is true for any element smaller than $A_{p}$ and consider $I=\left(11_{p}, A_{p}\right)$. Let $a$ and $b$ be the two largest elements of $A$. By induction for every element $X \in I$ with $\mu^{11}(X) \neq 0$ we have $X \leq a b 1_{p}$. Therefore, $L_{I}^{a b 1_{p}}$ is empty, which implies $\mu^{11}\left(A_{p}\right)=0$.

Lemma 4.6. For any $a \geq b>1$ :

$$
\mu^{11}\left(a b 1_{p}\right)=-\mu^{11}\left(a b_{p}\right)
$$

Proof. Figure 4.1 shows that $\mu^{11}\left(221_{p}\right)=-\mu^{11}\left(22_{p}\right)=0$. Assume the claim is true for any $i j_{p}<a b_{p}$ and consider the interval $I=\left(11_{p}, a b 1_{p}\right)$. Let $Z=\{a, b-1,1\}_{p}$, by Lemma 4.5 we know that the elements of $L_{I}^{Z}$ are:

$$
L_{I}^{Z}=\left\{i b 1_{p} \mid b \leq i<a\right\} \cup\left\{j b_{p} \mid b \leq j \leq a\right\} .
$$


The induction hypothesis implies that $\mu^{11}\left(j b_{p}\right)+\mu^{11}\left(j b 1_{p}\right)=0$, for $b \leq j<a$. Therefore, $\mu^{11}\left(a b 1_{p}\right)=-\mu^{11}\left(a b_{p}\right)$.

Lemma 4.7. For any $a-1>b>1$ :

$$
\mu^{11}\left(a b_{p}\right)=-\mu^{11}\left(\{a-1, b\}_{p}\right) .
$$

Proof. We can see in Figure 4.1 that $\mu^{11}\left(42_{p}\right)=-\mu^{11}\left(32_{p}\right)$. Assume the claim is true for $i j_{p}<a b_{p}$ and let $I=\left(11_{p}, a b_{p}\right)$. Let $Z=\{a, b-2,1\}_{p}$, by Lemma 4.5 the elements of $L_{I}^{Z}$ are of the form $i j_{p}$ or $i j 1_{p}$ and must have $i, j \geq b-1$. So:

$$
\begin{aligned}
L_{I}^{Z}= & \left\{\{a-1, b\}_{p},\{a-1, b-1\}_{p},\{a, b-1\}_{p}\right\} \\
& \cup\left\{\{i, j\}_{p},\{i, j, 1\}_{p} \mid b-1 \leq i \leq a-2, j \in\{b, b-1\}\right\}
\end{aligned}
$$

Lemma 4.6 and the induction hypothesis imply:

$$
\begin{aligned}
\mu^{11}\left(i j_{p}\right)+\mu^{11}\left(i j 1_{p}\right) & =0, \\
\mu^{11}\left(\{a-1, b-1\}_{p}\right)+\mu^{11}\left(\{a, b-1\}_{p}\right) & =0 .
\end{aligned}
$$

Therefore, $\mu^{11}\left(a b_{p}\right)=-\mu^{11}\left(\{a-1, b\}_{p}\right)$.

Proposition 4.8. For any $x>1$ :

$$
\mu^{11}\left(\{x, x-1\}_{p}\right)=-\mathcal{F}_{x-1} \quad \text { and } \quad \mu^{11}\left(x x_{p}\right)=\mathcal{F}_{x-2} .
$$

Proof. Figure 4.1 shows that $\mu^{11}\left(22_{p}\right)=\mathcal{F}_{0}, \mu^{11}\left(33_{p}\right)=\mathcal{F}_{1}, \mu^{11}\left(21_{p}\right)=-\mathcal{F}_{1}$ and $\mu^{11}\left(32_{p}\right)=-\mathcal{F}_{2}$. Assume the claim is true for any $i$ with $3<i<x$. Let $I=\left(11_{p},\{x, x-1\}_{p}\right)$ and $Z=\{x, x-3,1\}_{p}$, also let $J=\left(11_{p}, x x_{p}\right)$ and $K=\{x, x-2,1\}_{p}$. Lemma 4.5 allows us to compute the sets:

$$
\begin{aligned}
L_{I}^{Z}=\left\{\{x, x-2\}_{p},\{x-1, x-1\}_{p},\{x-1, x-2\}_{p},\{x-2, x-2\}_{p},\right. \\
\left.\{x-1, x-2,1\}_{p},\{x-2, x-2,1\}_{p}\right\}, \\
L_{J}^{K}=\left\{\{x, x-1\}_{p},\{x-1, x-1\}_{p}\right\} .
\end{aligned}
$$

By Lemma 4.6, Lemma 4.7 and the induction hypothesis we get

$$
\begin{aligned}
& \mu^{11}\left(\{x-1, x-2\}_{p}\right)+\mu^{11}\left(\{x-1, x-2,1\}_{p}\right)=0 \\
& \mu^{11}\left(\{x-2, x-2\}_{p}\right)+\mu^{11}\left(\{x-2, x-2,1\}_{p}\right)=0 \\
& \mu^{11}\left(\{x, x-2\}_{p}\right)=-\mu^{11}\left(\{x-1, x-2\}_{p}\right)=\mathcal{F}_{x-2} \\
& \mu^{11}\left(\{x-1, x-1\}_{p}\right)=\mathcal{F}_{x-3}
\end{aligned}
$$

Therefore,

$$
\begin{aligned}
\mu^{11}\left(\{x, x-1\}_{p}\right)=-\mu^{11}\left(\{x, x-2\}_{p}\right)-\mu^{11}\left(\{x-1, x-1\}_{p}\right) & =-\mathcal{F}_{x-2}-\mathcal{F}_{x-3} \\
& =-\mathcal{F}_{x-1}
\end{aligned}
$$

Moreover, Equation (4.1) then allows us to compute $\mu^{11}\left(x x_{p}\right)=-\mu^{11}\left(\{x, x-1\}_{p}\right)-\mu^{11}\left(\{x-1, x-1\}_{p}\right)=\mathcal{F}_{x-1}-\mathcal{F}_{x-3}=\mathcal{F}_{x-2}$ 
Combining Lemma 4.7 and Proposition 4.8 gives the following corollary:

Corollary 4.9. For any $a>b>1$ :

$$
\mu^{11}\left(a b_{p}\right)=(-1)^{a+b} \mathcal{F}_{b} .
$$

\subsection{The cases $1 \leq x \leq 5$}

To prove the results in this subsection we use discrete Morse theory, first developed in [For95]. In particular we use the methods introduced in [BH05] for applying discrete Morse theory to the order complex of a poset. We give a brief introduction to the necessary theory, but for further details we refer the reader to [SV06, Section 4]. In this subsection we consider the dual poset exclusively, so we let $\leq$ denote the dual partial order and drop the $*$ superscript. Also, we use $A, B$ and $C$ to denote chains and $a_{i}, b_{i}$ and $c_{i}$ to denote the $i$ 'th element of these chains, respectively.

Consider an interval $[x, y]$. We say two chains diverge at index $i$ if they agree up to $i$ but the $i+1$ 'th elements are different. An ordering $\triangleleft$ of the maximal chains of $(x, y)$ is a $P L$-ordering if given two chains $A \triangleleft B$ which diverge at $i$ and two chains $A^{\prime}$ and $B^{\prime}$ which agree to index $i+1$ with $A$ and $B$, respectively, then $A^{\prime} \triangleleft B^{\prime}$.

Let $C \backslash \hat{C}$ be the chain obtained by deleting $\hat{C}$ from $C$. Consider a PLordering $\triangleleft$ of the maximal chains of $(x, y)$, along with a maximal chain $C$ and subchain $\left(c_{i}, c_{i+k+1}\right)_{C}:=\left(c_{i+1}<c_{i+2}<\cdots<c_{i+k}\right)$. If $C \backslash\left(c_{i}, c_{i+k+1}\right)_{C}$ is a subchain of a chain $B \triangleleft C$, then we say $\left(c_{i}, c_{i+k+1}\right)$ is a skipped interval of $C$, of size $k$. A skipped interval is a minimal skipped interval (MSI) if it does not strictly contain another skipped interval.

Let $I_{1}, I_{2}, \ldots, I_{t}$ be the MSIs of a chain $C$ in increasing order of the index of their first element. Set $J_{1}=I_{1}$, and then $I_{k}^{\prime}=I_{k} \backslash J_{1}$, for all $k>1$, remove any $I_{k}^{\prime}$ which is no longer minimal, and set the first remaining one as $J_{2}$. Repeat this process until there are no non-empty modified MSIs remaining, and denote the set of $J_{1}, J_{2}, \ldots, J_{k^{\prime}}$ by $\mathcal{J}(C)$. A chain $C$ is critical if every element of $C$ appears in at least one element of $\mathcal{J}(C)$.

Given a PL-ordering and the sets $\mathcal{J}(C)$ we can compute the Möbius function $\mu(x, y)$ which equals the number of critical chains in any PL-ordering, with the sign given by the rank, see [BH05, Proposition 3.1]. Another useful result is [BH05, Proposition 4.2] which states that a PL-ordering is a shelling order if for every chain $C$ the set $\mathcal{J}(C)$ only contains skipped intervals of size 1 .

To apply discrete Morse theory we need to introduce a PL-ordering for the chains of $\mathcal{P}_{<5}^{*}$. Consider two graphs $\alpha_{p} \lessdot \beta_{p}$ in $\mathcal{P}^{*}$, to get from $\alpha_{p}$ to $\beta_{p}$ we must delete a vertex of $\alpha_{p}$ from a path of length $u \geq 1$. Deleting this point will take the component $u_{p}$ to $w^{1} w^{2}{ }_{p}$, where $w^{1}+w^{2}=u-1$ and $w^{1} \geq w^{2} \geq 0$. Define the operation between $\alpha_{p}$ and $\beta_{p}$ as the pair $u$ and $w:=\left\{w^{1}, w^{2}\right\}$ which we call the domain and image of the operation, respectively. We denote the operation by $\left(\begin{array}{c}u \\ w^{1} w^{2}\end{array}\right)_{p}$, or as $\left(\begin{array}{c}u \\ w_{1}\end{array}\right)_{p}$ when $w_{2}=0$. 
We can represent any maximal chain by the operations applied at each step. Given a maximal chain $C=c_{1}<c_{2}<\cdots<c_{n}$, of an interval of $\mathcal{P}^{*}$, define the operation chain $\Lambda(C)=\left(\lambda_{1}^{C}, \lambda_{2}^{C}, \ldots, \lambda_{n-1}^{C}\right)$, where $\lambda_{i}^{C}$ is the operation that takes $c_{i}$ to $c_{i+1}$. For example, $C=51_{p}<221_{p}<211_{p}<21_{p}$ has the operation chain $\Lambda(C)=\left(\left(\begin{array}{c}5 \\ 22\end{array}\right)_{\mathrm{p}},\left(\begin{array}{l}2 \\ 1\end{array}\right)_{\mathrm{p}},\left(\begin{array}{l}1 \\ 0\end{array}\right)_{\mathrm{p}}\right)$. Note that knowing the operation chain $\Lambda(C)$ and at least one element of $C$ uniquely determines $C$.

We can define a PL-ordering of the maximal chains of any interval of $\mathcal{P}^{*}$ by defining an ordering $\prec$ on the set of all possible operations. To do this take two maximal chains $B$ and $C$ which diverge at $i$ and order $B$ before $C$, denoted $B \triangleleft C$, if and only if $\lambda_{i}^{B} \prec \lambda_{i}^{C}$. We can see from Figure 4.2 that there are 9 possible operations in $\mathcal{P}_{\leq 5}^{*}$, which we give the following order:

$$
\left(\begin{array}{c}
5 \\
22
\end{array}\right)_{\mathrm{p}} \prec\left(\begin{array}{c}
4 \\
21
\end{array}\right)_{\mathrm{p}} \prec\left(\begin{array}{c}
3 \\
2
\end{array}\right)_{\mathrm{p}} \prec\left(\begin{array}{c}
5 \\
4
\end{array}\right)_{\mathrm{p}} \prec\left(\begin{array}{c}
5 \\
31
\end{array}\right)_{\mathrm{p}} \prec\left(\begin{array}{c}
4 \\
3
\end{array}\right)_{\mathrm{p}} \prec\left(\begin{array}{c}
3 \\
11
\end{array}\right)_{\mathrm{p}} \prec\left(\begin{array}{c}
1 \\
0
\end{array}\right)_{\mathrm{p}} \prec\left(\begin{array}{l}
2 \\
1
\end{array}\right)_{\mathrm{p}} .
$$

We choose this particular ordering as it seems to produce the simplest MSI's, thus allowing us to analyse the critical chains. Before proceeding further let us consider an example application of Discrete Morse Theory using our PLordering.

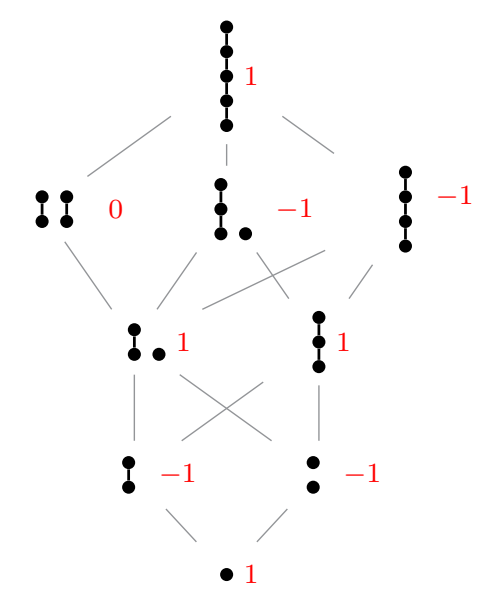

Figure 4.2: The interval $\left[1_{p}^{1}, 5_{p}^{1}\right]$ in $\mathcal{G}$, with $\mu\left(1_{p}^{1}, X\right)$ in red.

Example 4.10. Consider the interval $\left(1_{p}^{1}, 5_{p}^{1}\right)$ from Figure 4.2. Below we can see the maximal chains of this interval in our given PL-ordering. To the right of each chain $C$ we have the set $\mathcal{J}(C)$. We can see that the final chain is critical 
as every element appears in an element of $\mathcal{J}(C)$, so we get that $\mu\left(1_{p}^{1}, 5_{p}^{1}\right)=1$.

$$
\begin{array}{ll}
22_{p}>21_{p}>2_{p} & :\{\} \\
22_{p}>21_{p}>11_{p} & :\left\{\left\{11_{p}\right\}\right\} \\
4_{p}>21_{p}>2_{p} & :\left\{\left\{4_{p}\right\}\right\} \\
4_{p}>21_{p}>11_{p} & :\left\{\left\{4_{p}\right\},\left\{11_{p}\right\}\right\} \\
4_{p}>3_{p}>2_{p} & :\left\{\left\{3_{p}\right\}\right\} \\
4_{p}>3_{p}>11_{p} & :\left\{\left\{3_{p}\right\},\left\{11_{p}\right\}\right\} \\
31_{p}>21_{p}>2_{p} & :\left\{\left\{31_{p}\right\}\right\} \\
31_{p}>21_{p}>11_{p} & :\left\{\left\{31_{p}\right\},\left\{11_{p}\right\}\right\} \\
31_{p}>111_{p}>11_{p} & :\left\{\left\{111_{p}\right\}\right\} \\
31_{p}>3_{p}>2_{p} & :\left\{\left\{31_{p}\right\},\left\{3_{p}\right\}\right\} \\
31_{p}>3_{p}>11_{p} & :\left\{\left\{31_{p}\right\},\left\{3_{p}\right\},\left\{11_{p}\right\}\right\}
\end{array}
$$

In Table 2 we represent all 81 possible pairs of operations $\lambda_{i}^{C}, \lambda_{i+1}^{C}$, where each cell of the table is split into three parts. The top part of the cell contains the chain $c_{i}<c_{i+1}<c_{i+2}$, where $c_{i}$ contains exactly the domains of $\lambda_{i}^{C}$ and $\lambda_{i+1}^{C}$, and $c_{i+1}<c_{i+2}$ is obtained by applying $\lambda_{i}^{C}$ and $\lambda_{i+1}^{C}$ to $c_{i}$. If the domain of $\lambda_{i+1}^{C}$ is contained in the image of $\lambda_{i}^{C}$, then there is a second chain where $c_{i}$ is exactly the domain of $\lambda_{i}^{C}$. The middle part of the cell contains all elements $b_{1}, \ldots, b_{k}$ that can be obtained from $c_{i}$ by operations ordered before $\lambda_{i}^{C}$. The bottom part of the cell contains the operations $O_{1}, \ldots, O_{k}$, where $O_{j}$ maps $b_{j}$ to $c_{i+2}$. If no such operation exists then $O_{j}=\emptyset$.

Table 2 allows us to see all MSIs of size 1, but there are larger MSIs (which do not appear in the table), such as $\left(53_{p}, 32_{p}\right)$ which is an MSI of size 2 of the chain $53_{p}<52_{p}<42_{p}<32_{p}$. Therefore, this PL-ordering is not a shelling order. In fact, there is no ordering of the operations which induces a shelling order in this way, that is, for every ordering we can find a MSI of size greater than one. We have computationally verified this, the smallest interval needed to show it is $\left[543_{p}, 111_{p}\right]^{2}$. However, this does not imply that the intervals of $\mathcal{P}_{<5}^{*}$ are not shellable. In fact, due to the highly structured nature of these intervals, we believe it is likely they are all shellable, but a different approach is needed to prove this.

To show the Möbius function results we need to count the critical chains, so first we establish some notation and present some results on what can be an MSI. We say that $C=c_{i}<c_{i+1}<\cdots<c_{i+k+1}$ contains the operation $\left(\begin{array}{l}u \\ w\end{array}\right)_{p}$ if it appears in the operation chain $\Lambda(C)$. We say that $C$ begins or ends with $\left(\begin{array}{c}u \\ w\end{array}\right)_{p}$ if $\lambda_{i}^{C}=\left(\begin{array}{c}u \\ w\end{array}\right)_{p}$ or $\lambda_{i+k}^{C}=\left(\begin{array}{c}u \\ w\end{array}\right)_{p}$, respectively. Similarly, we say a skipped interval $\left(c_{i}, c_{j}\right)$ contains, begins or ends with an operation if the subchain $c_{i+1}<\cdots<c_{j-1}$ has that property. We denote by $C\left(\begin{array}{l}u \\ w\end{array}\right)_{p}$ the number of times $\left(\begin{array}{l}u \\ w\end{array}\right)_{p}$ appears in $\Lambda(C)$. We can construct a new chain $A$ by changing the operations in $\Lambda(C)$ to get a

${ }^{2}$ This code is available upon request. 


\begin{tabular}{|c|c|c|c|c|c|c|c|c|c|}
\hline$\lambda_{i}^{C} \lambda_{i+1}^{C}$ & $\left(\begin{array}{c}5 \\
22\end{array}\right)$ & $\left(\begin{array}{c}4 \\
21\end{array}\right)$ & $\left(\begin{array}{l}3 \\
2\end{array}\right)$ & $\left(\begin{array}{l}5 \\
4\end{array}\right)$ & $\left(\begin{array}{c}5 \\
31\end{array}\right)$ & $\left(\begin{array}{l}4 \\
3\end{array}\right)$ & $\left(\begin{array}{l}3 \\
11\end{array}\right)$ & $\left(\begin{array}{l}1 \\
0\end{array}\right)$ & $\left(\begin{array}{l}2 \\
1\end{array}\right)$ \\
\hline \multirow{2}{*}{$\left(\begin{array}{c}5 \\
22\end{array}\right)$} & $55<522<2222$ & $54<422<2221$ & $53<322<222$ & $55<522<422$ & $55<522<3221$ & $54<422<322$ & $53<322<2211$ & $51<221<22$ & $\begin{array}{c}52<222<221 \\
5<22<21\end{array}$ \\
\hline & & & & & & & & & \\
\hline \multirow{3}{*}{$\left(\begin{array}{c}4 \\
21\end{array}\right)$} & $54<521<2221$ & $44<421<2211$ & $43<321<221$ & $54<521<421$ & $54<521<3211$ & $44<421<321$ & $43<321<2111$ & $\begin{aligned} 41<211 & <21 \\
4 & <21<2\end{aligned}$ & $\begin{array}{c}42<221<211 \\
4<21<11\end{array}$ \\
\hline & 422 & & & 422 & 422 & & & & \\
\hline & $\left(\begin{array}{l}4 \\
21\end{array}\right)$ & & & $\left(\begin{array}{l}2 \\
1\end{array}\right)$ & $\emptyset$ & & & & \\
\hline \multirow{3}{*}{$\left(\begin{array}{l}3 \\
2\end{array}\right)$} & $53<52<222$ & $43<42<221$ & $33<32<22$ & $53<52<42$ & $53<52<321$ & $43<42<32$ & $33<32<211$ & $31<21<2$ & $\begin{array}{c}32<22<21 \\
3<2<1\end{array}$ \\
\hline & 322 & 321 & & 322 & 322 & 321 & & & \\
\hline & $\left(\begin{array}{l}3 \\
2\end{array}\right)$ & $\left(\begin{array}{l}3 \\
2\end{array}\right)$ & & $\emptyset$ & $\left(\begin{array}{l}2 \\
1\end{array}\right)$ & $\left(\begin{array}{l}1 \\
0\end{array}\right)$ & & & \\
\hline \multirow{3}{*}{$\left(\begin{array}{l}5 \\
4\end{array}\right)$} & $55<54<422$ & $\begin{array}{c}54<44<421 \\
5<4<21\end{array}$ & $53<43<42$ & $55<54<44$ & $55<54<431$ & $\begin{array}{c}54<44<43 \\
5<4<3\end{array}$ & $53<43<411$ & $51<41<4$ & $52<42<41$ \\
\hline & 522 & 422,521 & 322,52 & 522 & 522 & $\begin{array}{c}422,521 \\
22\end{array}$ & 322,52 & 221 & 222 \\
\hline & $\left(\begin{array}{l}5 \\
4\end{array}\right)$ & $\begin{array}{c}\left(\begin{array}{l}2 \\
1\end{array}\right),\left(\begin{array}{l}5 \\
4\end{array}\right) \\
\left(\begin{array}{l}2 \\
1\end{array}\right)\end{array}$ & $\emptyset,\left(\begin{array}{l}5 \\
4\end{array}\right)$ & $\emptyset$ & $\emptyset$ & 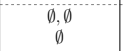 & $\emptyset, \emptyset$ & $\emptyset$ & $\emptyset$ \\
\hline \multirow{3}{*}{$\left(\begin{array}{l}5 \\
31\end{array}\right)$} & $55<531<3221$ & $54<431<3211$ & $\begin{array}{c}53<331<321 \\
5<31<21\end{array}$ & $55<531<431$ & $55<531<3311$ & $54<431<331$ & $\begin{array}{c}53<331<3111 \\
5<31<111\end{array}$ & $\begin{array}{c}51<311<31 \\
5<31<3\end{array}$ & $52<321<311$ \\
\hline & 522,54 & $422,521,44$ & $\begin{array}{c}322,52,43 \\
22,4\end{array}$ & 522,54 & 522,54 & $422,521,44$ & $\begin{array}{c}322,52,43 \\
22,4\end{array}$ & $\begin{array}{c}221,41 \\
22,4\end{array}$ & 222,42 \\
\hline & $\left(\begin{array}{l}5 \\
31\end{array}\right), \emptyset$ & $\emptyset,\left(\begin{array}{l}5 \\
31\end{array}\right), \emptyset$ & $\begin{array}{c}\left(\begin{array}{c}2 \\
1\end{array}\right),\left(\begin{array}{l}5 \\
31\end{array}\right),\left(\begin{array}{l}41 \\
21\end{array}\right) \\
\left(\begin{array}{l}2 \\
1\end{array}\right),\left(\begin{array}{l}4 \\
21\end{array}\right)\end{array}$ & $\emptyset,\left(\begin{array}{l}5 \\
31\end{array}\right)$ & $\emptyset, \emptyset$ & $\emptyset, \emptyset, \emptyset$ & $\begin{array}{c}\emptyset, \emptyset, \emptyset \\
\emptyset, \emptyset\end{array}$ & $\begin{array}{l}\emptyset,\left(\begin{array}{l}4 \\
3\end{array}\right) \\
\emptyset,\left(\begin{array}{l}4 \\
3\end{array}\right)\end{array}$ & $\emptyset, \emptyset$ \\
\hline \multirow{3}{*}{$\left(\begin{array}{l}4 \\
3\end{array}\right)$} & $54<53<322$ & $44<43<321$ & \begin{tabular}{|c|}
$43<33<32$ \\
$4<3<2$ \\
\end{tabular} & $54<53<43$ & $54<53<331$ & $44<43<33$ & $\begin{array}{c}43<33<311 \\
4<3<11\end{array}$ & $41<31<3$ & $42<32<31$ \\
\hline & $422,521,44,431$ & 421 & $\begin{array}{c}321,42 \\
21\end{array}$ & $422,44,521,431$ & $422,521,44,431$ & 421 & $\begin{array}{c}321,42 \\
21\end{array}$ & 211 & 221 \\
\hline & $\left(\begin{array}{l}4 \\
3\end{array}\right), \emptyset, \emptyset, \emptyset$ & $\left(\begin{array}{l}4 \\
3\end{array}\right)$ & $\begin{array}{c}\left(\begin{array}{c}1 \\
0\end{array}\right),\left(\begin{array}{l}1 \\
3\end{array}\right) \\
\left(\begin{array}{l}1 \\
0\end{array}\right)\end{array}$ & $\emptyset,\left(\begin{array}{l}4 \\
3\end{array}\right), \emptyset,\left(\begin{array}{l}1 \\
0\end{array}\right)$ & $\emptyset, \emptyset, \emptyset,\left(\begin{array}{l}4 \\
3\end{array}\right)$ & $\emptyset$ & $\begin{array}{c}\left(\begin{array}{l}2 \\
1\end{array}\right),(0) \\
\left(\begin{array}{l}2 \\
1\end{array}\right)\end{array}$ & $\emptyset$ & $\emptyset$ \\
\hline \multirow{3}{*}{$\left(\begin{array}{c}3 \\
11\end{array}\right)$} & $53<511<2211$ & $43<411<2111$ & $33<311<211$ & $53<511<411$ & $53<511<3111$ & $43<411<311$ & $33<311<1111$ & $\begin{array}{c}31<111<11 \\
3<11<1\end{array}$ & $32<211<111$ \\
\hline & $322,52,43,331$ & $321,42,33$ & 32 & $322,52,43,331$ & $322,52,43,331$ & $321,42,33$ & 32 & $\begin{array}{c}21 \\
2\end{array}$ & 22 \\
\hline & $\left(\begin{array}{l}3 \\
11\end{array}\right), \emptyset, \emptyset, \emptyset$ & $\left(\begin{array}{l}3 \\
11\end{array}\right), \emptyset, \emptyset$ & $\left(\begin{array}{l}3 \\
11\end{array}\right)$ & $\emptyset, \emptyset,\left(\begin{array}{l}3 \\
11\end{array}\right), \emptyset$ & $\emptyset, \emptyset, \emptyset,\left(\begin{array}{l}3 \\
11\end{array}\right)$ & $\left(\begin{array}{l}2 \\
1\end{array}\right), \emptyset,\left(\begin{array}{l}3 \\
11\end{array}\right)$ & $\emptyset$ & $\left.\begin{array}{l}2 \\
1 \\
(2 \\
1\end{array}\right)$ & $\emptyset$ \\
\hline \multirow{3}{*}{$\left(\begin{array}{l}1 \\
0\end{array}\right)$} & $51<5<22$ & $41<4<21$ & $31<3<2$ & $51<5<4$ & $51<5<31$ & $41<4<3$ & $31<3<11$ & $11<1<0$ & $21<2<1$ \\
\hline & $221,41,311$ & 211,31 & 21,111 & $221,41,311$ & $221,41,311$ & 211,31 & 21,111 & & \\
\hline & $\left(\begin{array}{l}1 \\
0\end{array}\right), \emptyset, \emptyset$ & $\left(\begin{array}{l}1 \\
0\end{array}\right),\left(\begin{array}{l}3 \\
2\end{array}\right)$ & $\left(\begin{array}{l}1 \\
0\end{array}\right), \emptyset$ & $\emptyset,\left(\begin{array}{l}1 \\
0\end{array}\right), \emptyset$ & $\emptyset,\left(\begin{array}{l}4 \\
3\end{array}\right),\left(\begin{array}{l}1 \\
0\end{array}\right)$ & $\emptyset,\left(\begin{array}{l}1 \\
0\end{array}\right)$ & $\left(\begin{array}{l}2 \\
1\end{array}\right),\left(\begin{array}{l}1 \\
0\end{array}\right)$ & & \\
\hline \multirow{3}{*}{$\left(\begin{array}{l}2 \\
1\end{array}\right)$} & $52<51<221$ & $42<41<211$ & $32<31<21$ & $52<51<41$ & $52<51<311$ & $42<41<31$ & $32<31<111$ & $\begin{array}{c}21<11<1 \\
2<1<0\end{array}$ & $22<21<11$ \\
\hline & $222,42,321$ & 221,32 & 22,211 & $222,42,321$ & $222,42,321$ & 221,32 & 22,211 & 2 & \\
\hline & $\left(\begin{array}{l}2 \\
1\end{array}\right),\left(\begin{array}{l}4 \\
21\end{array}\right),\left(\begin{array}{l}3 \\
2\end{array}\right)$ & $\left(\begin{array}{l}2 \\
1\end{array}\right),\left(\begin{array}{l}3 \\
11\end{array}\right)$ & $\left(\begin{array}{l}2 \\
1\end{array}\right),\left(\begin{array}{l}1 \\
0\end{array}\right)$ & $\emptyset,\left(\begin{array}{l}2 \\
1\end{array}\right), \emptyset$ & $\emptyset, \emptyset,\left(\begin{array}{l}2 \\
1\end{array}\right)$ & $\emptyset,\left(\begin{array}{l}2 \\
1\end{array}\right)$ & $\emptyset,\left(\begin{array}{l}2 \\
1\end{array}\right)$ & $\left(\begin{array}{l}2 \\
1\end{array}\right)$ & \\
\hline
\end{tabular}

Table 2: The table of all pairs $\left(\lambda_{i}^{C}, \lambda_{i+1}^{C}\right)$ where each cell is split into three parts. The top part is $c_{i}<c_{i+1}<c_{i+2}$. The middle part contains all elements $b_{1}, \ldots, b_{k}$ that can be obtained from $c_{i}$ by operations ordered before $\lambda_{i}^{C}$. The bottom part contains the operations $O_{1}, \ldots, O_{k}$, where $O_{j}$ maps $b_{j}$ to $c_{i+2}$, if no such operation exists then $O_{j}=\emptyset$. So $\left(c_{i}, c_{i+2}\right)$ is an MSI if and only if the cell $\left(\lambda_{i}^{C}, \lambda_{i+1}^{C}\right)$ has at least one operation $O \neq \emptyset$ in the bottom part of the cell. We omit the subscript $p$ in the table for ease of notation. 
new operation chain $\Lambda(A)$, but we must ensure that $\Lambda(A)$ is a valid operation chain. An operation chain $\Lambda(A)$ is invalid if $\lambda_{i}^{A}=\left(\begin{array}{c}u \\ w\end{array}\right)_{p}$ and there is no $u$ in $a_{i}$. Note that if $I$ is a skipped interval of $C$ then $I$ is a skipped interval of any chain $D$ which contains $C$, so when looking to see if $I=\left(c_{i}, c_{j}\right)$ appears as an MSI of a chain in $\mathcal{P}_{\leq 5}^{*}$ it suffices to consider chains from $c_{i}$ to $c_{j}$.

Lemma 4.11. If $I=\left(c_{i}, c_{i+k+1}\right)$ is a skipped interval of the chain $C=c_{i}<$ $c_{i+1}<\cdots<c_{i+k+1}$ in $\mathcal{P}_{\leq 5}^{*}$, with $k>1$, and $B \triangleleft C$ is a chain from $c_{i}$ to $c_{i+k+1}$, then $I$ is not minimal if any of the following hold:

1. $C$ contains a pair $\lambda_{y}^{C} \prec \lambda_{x}^{C}$, where $x<y$ and $\left(\lambda_{y}^{C}, \lambda_{x}^{C}\right) \neq\left(\left(\begin{array}{l}1 \\ 0\end{array}\right)_{\mathrm{p}},\left(\begin{array}{l}2 \\ 1\end{array}\right)_{\mathrm{p}}\right)$,

2. Either of $C$ or $B$ contain both $\left(\begin{array}{c}3 \\ 11\end{array}\right)_{\mathrm{p}}$ and $\left(\begin{array}{l}1 \\ 0\end{array}\right)_{\mathrm{p}}$,

3. $C$ contains both $\left(\begin{array}{c}5 \\ 31\end{array}\right)_{\mathrm{p}}$ and $\left(\begin{array}{l}1 \\ 0\end{array}\right)_{\mathrm{p}}$

4. B contains $\left(\begin{array}{l}2 \\ 1\end{array}\right)_{\mathrm{p}}$ and $\lambda_{i+k}^{C}=\left(\begin{array}{l}2 \\ 1\end{array}\right)_{\mathrm{p}}$,

5. $B$ contains $\lambda_{i}^{C}$.

Proof. Part (1): If there is a pair $x<y$ with $\lambda_{y}^{C} \prec \lambda_{x}^{C}$ and $\left(\lambda_{y}^{C}, \lambda_{x}^{C}\right) \neq$ $\left(\left(\begin{array}{l}1 \\ 0\end{array}\right)_{\mathrm{p}},\left(\begin{array}{l}2 \\ 1\end{array}\right)_{\mathrm{p}}\right)$, then there is a pair $\lambda_{\ell+1}^{C} \prec \lambda_{\ell}^{C}$, with $x \leq \ell<y$ and $\left(\lambda_{\ell}^{C}, \lambda_{\ell+1}^{C}\right) \neq$ $\left(\left(\begin{array}{l}1 \\ 0\end{array}\right)_{\mathrm{p}},\left(\begin{array}{l}2 \\ 1\end{array}\right)_{\mathrm{p}}\right)$. However, Table 2 shows that for any such $\lambda_{\ell+1}^{C} \prec \lambda_{\ell}^{C}$, that is, everything south east of the diagonal except $\left(\left(\begin{array}{l}1 \\ 0\end{array}\right)_{\mathrm{p}},\left(\begin{array}{l}2 \\ 1\end{array}\right)_{\mathrm{p}}\right)$, we get an MSI $\left(c_{\ell}, c_{\ell+2}\right)$. So $I$ is not minimal.

Part (2): Suppose $C$ contains $\left(\begin{array}{c}3 \\ 11\end{array}\right)_{\mathrm{p}}$ and $\left(\begin{array}{l}1 \\ 0\end{array}\right)_{\mathrm{p}}$, with $\lambda_{x}^{C}=\left(\begin{array}{c}3 \\ 11\end{array}\right)_{\mathrm{p}}$ and $\lambda_{y}^{C}=\left(\begin{array}{l}1 \\ 0\end{array}\right)_{\mathrm{p}}$ the leftmost occurrence of each. If $y<x$, then $I$ is not minimal by (1). If $x<y$, then we can create a new chain from $c_{x}$ to $c_{y+1}$ with the operation chain $\Lambda(A)=\left(\begin{array}{l}3 \\ 2\end{array}\right)_{\mathrm{p}}, \lambda_{x+1}^{C}, \lambda_{x+2}^{C}, \ldots, \lambda_{y-1}^{C},\left(\begin{array}{l}2 \\ 1\end{array}\right)_{\mathrm{p}}$. We can see that $\Lambda(A)$ is a valid operation chain because the domain of $\lambda_{x}^{C}$ and $\lambda_{x}^{A}$ are the same, and their images contain the domains of $\lambda_{y}^{C}$ and $\lambda_{y}^{A}$, respectively. So, $a_{y+1}=c_{y+1}$ and $A$ is lexicographically less than $c_{x}<\cdots<c_{y}$, thus $\left(c_{x}, c_{y}\right)$ is a skipped interval contained in $I$, so $I$ is not minimal.

Suppose $B$ contains $\left(\begin{array}{c}3 \\ 11\end{array}\right)_{\mathrm{p}}$ and $\left(\begin{array}{l}1 \\ 0\end{array}\right)_{\mathrm{p}}$. If $\left(\begin{array}{l}1 \\ 0\end{array}\right)_{\mathrm{p}}$ is before $\left(\begin{array}{c}3 \\ 11\end{array}\right)_{\mathrm{p}}$ in $\Lambda(B)$, then create a new operation chain $\Lambda(A)$ by moving $\left(\begin{array}{l}1 \\ 0\end{array}\right)_{\mathrm{p}}$ to immediately after $\left(\begin{array}{c}3 \\ 11\end{array}\right)_{\mathrm{p}}$. This is a valid operation chain as no operation acts on the image of $\left(\begin{array}{l}1 \\ 0\end{array}\right)_{\mathrm{p}}$. We can then apply the same argument to $\Lambda(A)$ as that used above, for the case $C$ contains both $\left(\begin{array}{c}3 \\ 11\end{array}\right)_{\mathrm{p}}$ and $\left(\begin{array}{l}1 \\ 0\end{array}\right)_{\mathrm{p}}$, to see that $I$ is not minimal.

Part (3): Suppose $C$ contains $\left(\begin{array}{c}5 \\ 31\end{array}\right)_{\mathrm{p}}$ and $\left(\begin{array}{l}1 \\ 0\end{array}\right)_{\mathrm{p}}$. We can apply an analogous argument to (2) where we create a new operation chain $\Lambda(A)$ from $\Lambda(C)$ by replacing $\left(\begin{array}{c}5 \\ 31\end{array}\right)_{\mathrm{p}}$ and $\left(\begin{array}{l}1 \\ 0\end{array}\right)_{\mathrm{p}}$ with $\left(\begin{array}{l}5 \\ 4\end{array}\right)_{\mathrm{p}}$ and $\left(\begin{array}{l}4 \\ 3\end{array}\right)_{\mathrm{p}}$, respectively.

Part (4): Suppose $B$ contains $\left(\begin{array}{l}2 \\ 1\end{array}\right)_{\mathrm{p}}$. Let $\Lambda(A)$ be obtained from $\Lambda(B)$ by moving $\lambda_{t}^{B}=\left(\begin{array}{l}2 \\ 1\end{array}\right)_{\mathrm{p}}$ to the end. If this creates an invalid chain then it must be caused by $\lambda_{x}^{B}=\left(\begin{array}{l}1 \\ 0\end{array}\right)_{\mathrm{p}}$, with $x>t$. So also move the $\left(\begin{array}{l}1 \\ 0\end{array}\right)_{\mathrm{p}}$ to the penultimate position of $\Lambda(A)$, which is valid as $c_{i+k+1}$ contains a 1 so there must be an operation $\lambda_{v}^{B}$ whose image contains a 1, with $v>k$. We can see that $A \triangleleft B \triangleleft C$, because we move $\left(\begin{array}{l}2 \\ 1\end{array}\right)_{\mathrm{p}}$ to the right of an operation ordered before it. Also we know that $c_{i+k}=a_{i+k}$ since $\lambda_{i+k}^{C}=\lambda_{i+k}^{A}=\left(\begin{array}{l}2 \\ 1\end{array}\right)$ p. Therefore, $A$ implies that $\left(c_{i}, c_{i+k}\right)$ is a skipped interval, contradicting the claim that $I$ is minimal. 
Part (5): Suppose $\Lambda(B)$ contains $\lambda_{i}^{C}$. Let $\Lambda(A)$ be obtained from $\Lambda(B)$ by moving $\lambda_{i}^{C}$ to the start. Clearly $b_{i}=c_{i}$ contains the domain of $\lambda_{i}^{C}$ so this is a valid operation chain. By Part (1) we know that $\lambda_{i}^{C} \prec \lambda_{i+1}^{C}$ (or $\lambda_{i}^{C}=\lambda_{i+1}^{C}$ in which case apply the following argument to the first index for which they differ). Also since $B \triangleleft C$ we know that $\lambda_{i+1}^{A}=\lambda_{i}^{B} \prec \lambda_{i}^{C}$ so $\lambda_{i+1}^{A} \prec \lambda_{i+1}^{C}$, and $\lambda_{i}^{A}=\lambda_{i}^{C}$ by construction, which implies that $A \triangleleft C$. Therefore, $\left(c_{i+1}, c_{i+k+1}\right)$ is a skipped interval, so $I$ is not minimal.

Lemma 4.12. There is no MSI in any chain of $\mathcal{P}_{\leq 5}^{*}$ that ends in $\left(\begin{array}{l}2 \\ 1\end{array}\right)_{\mathrm{p}}$.

Proof. Let $I, B$ and $C$ be as defined in Lemma 4.11. For a contradiction assume that $\lambda_{i+k}^{C}=\left(\begin{array}{l}2 \\ 1\end{array}\right)_{\mathrm{p}}$. So Lemma 4.11(4) implies that $B$ does not contain $\left(\begin{array}{l}2 \\ 1\end{array}\right)_{\mathrm{p}}$. There must be an operation in $C$ whose image contains 2, otherwise the number of 2's in $c_{i+k+1}$ and $b_{i+k+1}$ will differ, but we know that $c_{i+k+1}=b_{i+k+1}$. So $C$ must contain at least one of $\left(\begin{array}{c}5 \\ 22\end{array}\right)_{\mathrm{p}},\left(\begin{array}{c}4 \\ 21\end{array}\right)_{\mathrm{p}}$ or $\left(\begin{array}{l}3 \\ 2\end{array}\right)_{\mathrm{p}}$. In Claims 1 to 3 we show that $C$ cannot contain any of these, so we get a contradiction.

Claim 1. The chain $C$ cannot contain $\left(\begin{array}{c}5 \\ 22\end{array}\right)_{\mathrm{p}}$.

Proof. Suppose $C$ contains $\left(\begin{array}{c}5 \\ 22\end{array}\right)_{\mathrm{p}}$, then the leftmost occurrence is either at the start or is preceded by a different operation. If $\lambda_{i}^{C}=\left(\begin{array}{c}5 \\ 22\end{array}\right)_{\mathrm{p}}$, then $\lambda_{i}^{B}=\left(\begin{array}{c}5 \\ 22\end{array}\right)_{\mathrm{p}}$ otherwise $C \triangleleft B$, so $I$ is not minimal by Lemma 4.11(5). If $\left(\begin{array}{c}5 \\ 22\end{array}\right)_{\mathrm{p}}$ is preceded by a different operation $O$, then we must have $\left(\begin{array}{c}5 \\ 22\end{array}\right)_{\mathrm{p}} \prec O$ as $\left(\begin{array}{c}5 \\ 22\end{array}\right)_{\mathrm{p}}$ is the smallest label according to (4.2), so $I$ is not minimal by Lemma 4.11(1).

Claim 2. The chain $C$ cannot contain $\left(\begin{array}{c}4 \\ 21\end{array}\right)_{\mathrm{p}}$.

Proof. Suppose $C$ contains $\left(\begin{array}{c}4 \\ 21\end{array}\right)_{\mathrm{p}}$, and by Claim 1 we can assume $C$ does not contain $\left(\begin{array}{c}5 \\ 22\end{array}\right)_{\mathrm{p}}$. So $\lambda_{i}^{C}=\left(\begin{array}{c}4 \\ 21\end{array}\right)_{\mathrm{p}}$ because otherwise there is a pair $\left(\alpha,\left(\begin{array}{c}4 \\ 21\end{array}\right)_{\mathrm{p}}\right)$, with $\left(\begin{array}{c}4 \\ 21\end{array}\right)_{\mathrm{p}} \prec \alpha$, which implies $I$ is not minimal by Lemma 4.11(1). Moreover, $B$ cannot contain $\left(\begin{array}{c}4 \\ 21\end{array}\right)_{\mathrm{p}}$ by Lemma $4.11(5)$, so $\lambda_{i}^{B}=\left(\begin{array}{c}5 \\ 22\end{array}\right)_{\mathrm{p}}$ as $B \triangleleft C$. Therefore, $C$ must contain some operation whose domain is 5 , so either $\left(\begin{array}{c}5 \\ 4\end{array}\right)_{\mathrm{p}}$ or $\left(\begin{array}{c}5 \\ 31\end{array}\right)_{\mathrm{p}}$, otherwise the number of 5's in $c_{i+k+1}$ and $b_{i+k+1}$ will differ.

If $C$ contains $\left(\begin{array}{l}5 \\ 4\end{array}\right)_{\mathrm{p}}$, then the leftmost $\left(\begin{array}{l}5 \\ 4\end{array}\right)_{\mathrm{p}}$ must be preceded by $\left(\begin{array}{l}3 \\ 2\end{array}\right)_{\mathrm{p}}$, otherwise $I$ is not minimal by Table 2 . So $\Lambda(C)$ must begin $\left(\begin{array}{c}4 \\ 21\end{array}\right){ }_{\mathrm{p}}^{u},\left(\begin{array}{c}3 \\ 2\end{array}\right)_{\mathrm{p}} v,\left(\begin{array}{c}5 \\ 4\end{array}\right)_{\mathrm{p}}$, where $u, v>0$ denote multiple consecutive operations. Let $\Lambda(A)$ be the chain obtained from $\Lambda(C)$ by replacing the leftmost $\left(\begin{array}{c}4 \\ 21\end{array}\right)_{\mathrm{p}}$ with $\left(\begin{array}{c}5 \\ 22\end{array}\right)_{\mathrm{p}}$ and $\left(\begin{array}{l}5 \\ 4\end{array}\right)_{\mathrm{p}}$ with $\left(\begin{array}{l}2 \\ 1\end{array}\right)_{\mathrm{p}}$. It is easy to verify that $\Lambda(A)$ a valid operation chain, and we can see that in both case we remove 5 and add 2 and 1 . So $A \triangleleft C$ and $a_{u+v+1}=c_{u+v+1}$, which implies $\left(c_{i}, c_{u+v+1}\right)$ is a skipped interval contained in $I$, so $I$ is not minimal

If $C$ contains $\left(\begin{array}{c}5 \\ 31\end{array}\right)_{\mathrm{p}}$, and does not contain $\left(\begin{array}{l}5 \\ 4\end{array}\right)_{\mathrm{p}}$, then $\Lambda(C)$ must begin with $\left(\begin{array}{l}4 \\ 21\end{array}\right)_{\mathrm{p}}{ }^{u},\left(\begin{array}{l}5 \\ 31\end{array}\right)_{\mathrm{p}}$. By Lemma $4.11(3)$ we know that $C$ cannot contain $\left(\begin{array}{l}1 \\ 0\end{array}\right)_{\mathrm{p}}$ and by Lemma 4.11(2) we know that $B$ cannot contain both $\left(\begin{array}{c}3 \\ 11\end{array}\right)_{\mathrm{p}}$ and $\left(\begin{array}{c}1 \\ 0\end{array}\right)_{\mathrm{p}}$. So we consider the two cases where $B$ does or does not contain $\left(\begin{array}{l}3 \\ 11\end{array}\right)_{\mathrm{p}}$. 
If $B$ does not contain $\left(\begin{array}{l}3 \\ 11\end{array}\right)$ p , then counting the number of 1's added and the number of additional components created in $B$ and $C$, gives the following equalities:

$$
\begin{aligned}
C\left(\begin{array}{l}
2 \\
1
\end{array}\right)_{\mathrm{p}}+2 C\left(\begin{array}{c}
3 \\
11
\end{array}\right)_{\mathrm{p}}+C\left(\begin{array}{c}
4 \\
21
\end{array}\right)_{\mathrm{p}}+C\left(\begin{array}{c}
5 \\
31
\end{array}\right)_{\mathrm{p}}=B\left(\begin{array}{c}
5 \\
31
\end{array}\right)_{\mathrm{p}}-B\left(\begin{array}{l}
1 \\
0
\end{array}\right)_{\mathrm{p}} \\
C\left(\begin{array}{c}
3 \\
11
\end{array}\right)_{\mathrm{p}}+C\left(\begin{array}{c}
4 \\
21
\end{array}\right)_{\mathrm{p}}+C\left(\begin{array}{c}
5 \\
31
\end{array}\right)_{\mathrm{p}}=B\left(\begin{array}{c}
5 \\
31
\end{array}\right)_{\mathrm{p}}-B\left(\begin{array}{l}
1 \\
0
\end{array}\right)_{\mathrm{p}}+B\left(\begin{array}{c}
5 \\
22
\end{array}\right)_{\mathrm{p}}
\end{aligned}
$$

Subtracting (4.4) from (4.3) implies $C\left(\begin{array}{c}3 \\ 11\end{array}\right)_{\mathrm{p}}+C\left(\begin{array}{l}2 \\ 1\end{array}\right)_{\mathrm{p}}=-B\left(\begin{array}{c}5 \\ 22\end{array}\right)_{\mathrm{p}}$, which we know is not possible as all values are non-negative and $B\left(\begin{array}{c}5 \\ 22\end{array}\right)_{\mathrm{p}}>0$.

If $B$ contains $\left(\begin{array}{l}3 \\ 11\end{array}\right)_{\mathrm{p}}$, so it does not contain $\left(\begin{array}{c}1 \\ 0\end{array}\right)_{\mathrm{p}}$ by Lemma $4.11(2)$, then counting the components added, the number of 1 's added, the number of 5 's removed and the number of 2's added in $B$ and $C$ implies:

$$
\begin{aligned}
\left.C\left(\begin{array}{c}
3 \\
1
\end{array}\right)\right)_{\mathrm{p}}+C\left(\begin{array}{c}
4 \\
22
\end{array}\right)_{\mathrm{p}}+C\left(\begin{array}{c}
5 \\
31
\end{array}\right)_{\mathrm{p}} & =B\left(\begin{array}{c}
5 \\
31
\end{array}\right)_{\mathrm{p}}+B\left(\begin{array}{c}
3 \\
11
\end{array}\right)_{\mathrm{p}}+B\left(\begin{array}{c}
5 \\
22
\end{array}\right)_{\mathrm{p}} \\
C\left(\begin{array}{l}
2 \\
1
\end{array}\right)_{\mathrm{p}}+2 C\left(\begin{array}{c}
3 \\
11
\end{array}\right)_{\mathrm{p}}+C\left(\begin{array}{c}
4 \\
21
\end{array}\right)_{\mathrm{p}}+C\left(\begin{array}{c}
5 \\
31
\end{array}\right)_{\mathrm{p}} & =B\left(\begin{array}{c}
5 \\
31
\end{array}\right)_{\mathrm{p}}+2 B\left(\begin{array}{c}
3 \\
11
\end{array}\right)_{\mathrm{p}} \\
C\left(\begin{array}{c}
5 \\
31
\end{array}\right)_{\mathrm{p}} & =B\left(\begin{array}{c}
5 \\
31
\end{array}\right)_{\mathrm{p}}+B\left(\begin{array}{c}
52 \\
22
\end{array}\right)_{\mathrm{p}}+B\left(\begin{array}{c}
5 \\
4
\end{array}\right)_{\mathrm{p}} \\
C\left(\begin{array}{c}
4 \\
21
\end{array}\right)_{\mathrm{p}}-C\left(\begin{array}{l}
2 \\
1
\end{array}\right)_{\mathrm{p}} & =B\left(\begin{array}{c}
3 \\
2
\end{array}\right)_{\mathrm{p}}+2 B\left(\begin{array}{c}
5 \\
22
\end{array}\right)_{\mathrm{p}}
\end{aligned}
$$

Combining the above equations, by $(4.8)+(4.6)+(4.7)-2(4.5)$, gives

$$
B\left(\begin{array}{c}
5 \\
22
\end{array}\right)_{\mathrm{p}}+B\left(\begin{array}{c}
3 \\
2
\end{array}\right)_{\mathrm{p}}+B\left(\begin{array}{c}
5 \\
4
\end{array}\right)_{\mathrm{p}}=0
$$

which again is impossible as $B\left(\begin{array}{c}5 \\ 22\end{array}\right)_{\mathrm{p}}>0$ and all values are non-negative.

Therefore, we cannot have $\left(\begin{array}{l}5 \\ 31\end{array}\right)_{\mathrm{p}}^{\mathrm{p}}$ in $C$. So there is no operation in $C$ whose domain is 5 , which implies $c_{i+k+1} \neq b_{i+k+1}$, hence $C$ cannot start with $\left(\begin{array}{c}4 \\ 21\end{array}\right)_{\mathrm{p}}$.

Claim 3. The chain $C$ cannot contain $\left(\begin{array}{l}3 \\ 2\end{array}\right)_{\mathrm{p}}$.

Proof. Assume $C$ contains $\left(\begin{array}{c}3 \\ 2\end{array}\right)_{\mathrm{p}}$ and does not contain $\left(\begin{array}{c}5 \\ 22\end{array}\right)_{\mathrm{p}}$ or $\left(\begin{array}{c}4 \\ 21\end{array}\right)_{\mathrm{p}}$. So $C$ must begin with $\left(\begin{array}{l}3 \\ 2\end{array}\right)_{\mathrm{p}}$ by Lemma 4.11(1) and (4.2). First we show that $C$ cannot contain $\left(\begin{array}{c}5 \\ 31\end{array}\right)_{\mathrm{p}}$. If $\left(\begin{array}{c}5 \\ 31\end{array}\right)$ p is in $\Lambda(C)$, then $\Lambda(C)$ must begin $\left.\left(\begin{array}{l}3 \\ 2\end{array}\right)_{\mathrm{p}}{ }^{u},\left(\begin{array}{l}5 \\ 4\end{array}\right)\right)_{\mathrm{p}}{ }^{v},\left(\begin{array}{c}5 \\ 31\end{array}\right)_{\mathrm{p}}$. Let $\Lambda(A)$ be obtained from $\Lambda(C)$ by replacing the leftmost occurrences of $\left(\begin{array}{l}3 \\ 2\end{array}\right)_{\mathrm{p}},\left(\begin{array}{l}5 \\ 4\end{array}\right)_{\mathrm{p}}$ and $\left(\begin{array}{c}5 \\ 31\end{array}\right)_{\mathrm{p}}$ with $\left(\begin{array}{c}5 \\ 22\end{array}\right)_{\mathrm{p}},\left(\begin{array}{l}2 \\ 1\end{array}\right)_{\mathrm{p}}$ and $\left(\begin{array}{l}5 \\ 4\end{array}\right)_{\mathrm{p}}$, respectively. It is straightforward to see that this is a valid operation chain. Moreover, $a_{i+u+v+1}=c_{i+u+v+1}$ and $A$ is a lexicographically less chain, thus $\left(c_{i}, c_{i+u+v+1}\right)$ is a skipped interval contained in $I$, so $I$ is not minimal.

We can also see that there cannot be a $\left(\begin{array}{l}4 \\ 3\end{array}\right)_{\mathrm{p}}$ in $C$. If there is then let $\Lambda(A)$ be obtained from $\Lambda(C)$ by changing $\left(\begin{array}{l}3 \\ 2\end{array}\right)_{\mathrm{p}}$ to $\left(\begin{array}{c}4 \\ 21\end{array}\right)_{\mathrm{p}}$ and $\left(\begin{array}{c}4 \\ 3\end{array}\right)_{\mathrm{p}}$ to $\left(\begin{array}{c}1 \\ 0\end{array}\right)_{\mathrm{p}}$. So $A$ is lexicographically less, thus $I$ is not minimal.

By Lemma 4.11 we can assume $B$ does not contain $\left(\begin{array}{l}3 \\ 2\end{array}\right)_{\mathrm{p}}$ nor both $\left(\begin{array}{l}3 \\ 11\end{array}\right)_{\mathrm{p}}$ and $\left(\begin{array}{l}1 \\ 0\end{array}\right)_{\mathrm{p}}$. If there is no $\left(\begin{array}{c}3 \\ 11\end{array}\right)_{\mathrm{p}}$ in $B$ then we must have at least as many 3 's in $b_{i+k+1}$ as in $b_{i}$. But this is not possible, as $a_{i}$ and $a_{i+k+1}$ have a different number of 3's because we remove a 3 from $c_{i}$ and have no way to create more as $C$ does not contain $\left(\begin{array}{c}5 \\ 31\end{array}\right)_{\mathrm{p}}$ nor $\left(\begin{array}{l}4 \\ 3\end{array}\right)_{\mathrm{p}}$. So there must be a $\left(\begin{array}{c}3 \\ 11\end{array}\right)_{\mathrm{p}}$ in $B$, which means there is no $\left(\begin{array}{l}1 \\ 0\end{array}\right)_{\mathrm{p}}$. This implies there must be a $\left(\begin{array}{l}3 \\ 11\end{array}\right)_{\mathrm{p}}$ in $C$ as $b_{i+k+1}$ has more 
components than $b_{i}$ so we need some operation in $C$ that creates components, and $\left(\begin{array}{c}3 \\ 11\end{array}\right)_{\mathrm{p}}$ is the only remaining option. So $C$ can contain $\left(\begin{array}{l}3 \\ 2\end{array}\right)_{\mathrm{p}},\left(\begin{array}{c}5 \\ 4\end{array}\right)_{\mathrm{p}},\left(\begin{array}{c}3 \\ 11\end{array}\right)_{\mathrm{p}}$ and $\left(\begin{array}{c}2 \\ 1\end{array}\right)_{\mathrm{p}}$. So counting the number of components added and 3 's removed from $B$ and $C$, we get:

$$
\begin{aligned}
C\left(\begin{array}{c}
3 \\
11
\end{array}\right)_{\mathrm{p}} & =B\left(\begin{array}{c}
3 \\
11
\end{array}\right)_{\mathrm{p}}+B\left(\begin{array}{c}
5 \\
31
\end{array}\right)_{\mathrm{p}}+B\left(\begin{array}{c}
5 \\
22
\end{array}\right)_{\mathrm{p}}+B\left(\begin{array}{c}
4 \\
21
\end{array}\right)_{\mathrm{p}}, \\
C\left(\begin{array}{l}
3 \\
2
\end{array}\right)_{\mathrm{p}}+C\left(\begin{array}{c}
3 \\
11
\end{array}\right)_{\mathrm{p}} & =B\left(\begin{array}{c}
3 \\
11
\end{array}\right)_{\mathrm{p}}-B\left(\begin{array}{c}
5 \\
31
\end{array}\right)_{\mathrm{p}}-B\left(\begin{array}{l}
4 \\
3
\end{array}\right)_{\mathrm{p}} .
\end{aligned}
$$

Subtracting (4.9) from (4.10), and rearranging, we get:

$$
B\left(\begin{array}{c}
5 \\
22
\end{array}\right)_{\mathrm{p}}+B\left(\begin{array}{c}
4 \\
21
\end{array}\right)_{\mathrm{p}}+2 B\left(\begin{array}{c}
5 \\
31
\end{array}\right)_{\mathrm{p}}+B\left(\begin{array}{c}
4 \\
3
\end{array}\right)_{\mathrm{p}}+C\left(\begin{array}{c}
3 \\
2
\end{array}\right)_{\mathrm{p}}=0
$$

which is impossible, as we know that $C\left(\begin{array}{l}3 \\ 2\end{array}\right)_{\mathrm{p}}>0$ and all values are non-negative. Therefore, $C$ cannot begin $\left(\begin{array}{l}3 \\ 2\end{array}\right)$ p .

Lemma 4.13. If an MSI of a chain in $\mathcal{P}_{\leq 5}^{*}$ begins with $\left(\begin{array}{c}5 \\ 4\end{array}\right)$, then it is of size 1 .

Proof. Let $I, B$ and $C$ be as defined in Lemma 4.11 and suppose $C$ begins with $\left(\begin{array}{l}5 \\ 4\end{array}\right)_{\mathrm{p}}$. The only way to create a 4 is by the operation $\left(\begin{array}{l}5 \\ 4\end{array}\right)_{\mathrm{p}}$ and by Lemma $4.11(5)$, we know that $B$ does not contain $\lambda_{i}^{C}=\left(\begin{array}{c}5 \\ 4\end{array}\right)_{\mathrm{p}}$. Therefore, the number of 4's in $b_{i+k+1}$ is weakly less than in $b_{i}$. Since $C$ begins with $\left(\begin{array}{l}5 \\ 4\end{array}\right)$ p in order for the number of 4's in $c_{i+k+1}$ to be weakly less than in $c_{i}$ the chain $C$ must contain some operation whose domain is 4 . By Lemma 4.11(1) this cannot be $\left(\begin{array}{l}4 \\ 21\end{array}\right)$, so must be $\left(\begin{array}{l}4 \\ 3\end{array}\right)_{\mathrm{p}}$. Therefore, $C$ cannot contain $\left(\begin{array}{l}3 \\ 11\end{array}\right)_{\mathrm{p}}$, because $\left(\left(\begin{array}{l}4 \\ 3\end{array}\right)_{\mathrm{p}},\left(\begin{array}{l}3 \\ 11\end{array}\right)_{\mathrm{p}}\right)$ is an MSI (see Table 2), and $C$ does not contain $\left(\begin{array}{l}3 \\ 2\end{array}\right)_{\mathrm{p}}$ because $\left(\begin{array}{l}3 \\ 2\end{array}\right)_{\mathrm{p}} \prec\left(\begin{array}{l}5 \\ 4\end{array}\right)_{\mathrm{p}}$, so we cannot remove 3's.

The possible operations with domain 5 in $C$ are $\left(\begin{array}{c}5 \\ 31\end{array}\right)_{\mathrm{p}}$ and $\left(\begin{array}{c}5 \\ 4\end{array}\right)_{\mathrm{p}}$. Moreover, for every $\left(\begin{array}{l}5 \\ 4\end{array}\right)_{\mathrm{p}}$ we then must have a $\left(\begin{array}{l}4 \\ 3\end{array}\right)_{\mathrm{p}}$. This implies that for every 5 removed from $c_{i}$ there is an extra 3 in $c_{i+k+1}$. However, $B$ begins with $\left(\begin{array}{c}5 \\ 22\end{array}\right)_{\mathrm{p}}$ so this cannot be the case in $B$. So no valid $B$ exists, giving a contradiction to $I$ being a skipped interval

We now have the necessary information on the MSIs of $\mathcal{P}_{<5}^{*}$ to compute the critical chains. Recall that the Möbius function of an interval $[H, G]$ is equal to the number of critical chains, with the sign given by the rank. So we can use this to prove the cases $1 \leq x \leq 5$ of Theorem 4.1.

Proposition 4.14. For any $n \geq 1$ :

$$
\mu\left(1_{p}^{n}, 5_{p}^{n}\right)=\mathcal{C}_{n} .
$$

Proof. To show the result we need to compute all critical chains of $\left[1_{p}^{n}, 5_{p}^{n}\right]$. By Lemma 4.12 and the bottom row of Table 2 we know that a critical chain $C$ cannot contain $\left(\begin{array}{l}2 \\ 1\end{array}\right)_{\mathrm{p}}$, hence $C$ also cannot contain $\left(\begin{array}{c}5 \\ 22\end{array}\right)_{\mathrm{p}},\left(\begin{array}{c}4 \\ 21\end{array}\right)_{\mathrm{p}}$ nor $\left(\begin{array}{c}3 \\ 2\end{array}\right)_{\mathrm{p}}$, as $1_{p}^{n}$ does not contain any 2's. Also note that $C$ cannot contain $\left(\begin{array}{l}5 \\ 4\end{array}\right)_{\mathrm{p}}$, because either $\left(\begin{array}{c}5 \\ 4\end{array}\right)_{\mathrm{p}}$ is in the middle of an MSI or at the end of one MSI and beginning of another. The 
second case is not possible because Lemma 4.13 and Table 2 imply in any MSI beginning with $\left(\begin{array}{l}5 \\ 4\end{array}\right)_{\mathrm{p}}$ it is followed by one of $\left(\begin{array}{c}5 \\ 22\end{array}\right)_{\mathrm{p}},\left(\begin{array}{c}4 \\ 21\end{array}\right)_{\mathrm{p}}$ or $\left(\begin{array}{l}3 \\ 2\end{array}\right)_{\mathrm{p}}$. The first case is not possible because Table 2 implies if $\left(\begin{array}{l}5 \\ 4\end{array}\right)$ is preceded by anything other than $\left(\begin{array}{c}5 \\ 22\end{array}\right)_{\mathrm{p}},\left(\begin{array}{c}4 \\ 21\end{array}\right)_{\mathrm{p}}$ or $\left(\begin{array}{l}3 \\ 2\end{array}\right)_{\mathrm{p}}$ then it creates an MSI which ends with $\left(\begin{array}{l}5 \\ 4\end{array}\right)_{\mathrm{p}}$. Therefore, $C$ also cannot contain $\left(\begin{array}{l}4 \\ 3\end{array}\right)$ as there is never a 4 in any element of the chain. So the only operations in $C$ are $\left(\begin{array}{l}1 \\ 0\end{array}\right)_{\mathrm{p}},\left(\begin{array}{c}5 \\ 31\end{array}\right)_{\mathrm{p}}$, and $\left(\begin{array}{c}3 \\ 11\end{array}\right)_{\mathrm{p}}$.

We can see that $C\left(\begin{array}{c}5 \\ 31\end{array}\right)_{\mathrm{p}}=n, C\left(\begin{array}{l}3 \\ 11\end{array}\right)_{\mathrm{p}}=n$ and $C\left(\begin{array}{l}1 \\ 0\end{array}\right)_{\mathrm{p}}=2 n$, in order to get from $5_{p}^{n}$ to $1_{p}^{n}$. Note that $\left(\begin{array}{l}1 \\ 0\end{array}\right)_{\mathrm{p}}$ cannot be preceded by another $\left(\begin{array}{l}1 \\ 0\end{array}\right)_{\mathrm{p}}$, as this cannot create an MSI. So $\left(\begin{array}{l}1 \\ 0\end{array}\right)_{\mathrm{p}}$ is directly preceded by $\left(\begin{array}{c}5 \\ 31\end{array}\right)_{\mathrm{p}}$ or $\left(\begin{array}{l}3 \\ 11\end{array}\right)_{\mathrm{p}}$ and as $C\left(\begin{array}{c}5 \\ 31\end{array}\right)_{\mathrm{p}}+C\left(\begin{array}{c}3 \\ 11\end{array}\right)_{\mathrm{p}}=C\left(\begin{array}{l}1 \\ 0\end{array}\right)_{\mathrm{p}}$ we know that every $\left(\begin{array}{c}5 \\ 31\end{array}\right)_{\mathrm{p}}$ and $\left(\begin{array}{c}3 \\ 11\end{array}\right)_{\mathrm{p}}$ is followed by $\left(\begin{array}{l}1 \\ 0\end{array}\right)_{\mathrm{p}}$, so we have no choice in the placement of $\left(\begin{array}{l}1 \\ 0\end{array}\right)_{\mathrm{p}}$. Secondly note that we can only apply $\left(\begin{array}{c}3 \\ 11\end{array}\right)$ if there is a 3 for it to be applied to, which implies that to the left of every $\left(\begin{array}{c}3 \\ 11\end{array}\right)_{\mathrm{p}}$ there is at least one more $\left(\begin{array}{c}5 \\ 31\end{array}\right)_{\mathrm{p}}$ than $\left(\begin{array}{c}3 \\ 11\end{array}\right)_{\mathrm{p}}$. Table 2 implies that any chain of this form is critical. Therefore, if we let $\left(\begin{array}{c}5 \\ 31\end{array}\right)_{\mathrm{p}}^{\mathrm{p}}$ denote an north east step and $\left(\begin{array}{c}3 \\ 11\end{array}\right)$, denote a south east step, then the critical chains are in bijection with the Dyck paths of length $2 n$. Finally, the rank is equal to $4 n$, so the sign is given by $(-1)^{4 n}=1$.

Example 4.15. In Example 4.10 we saw that the only critical chain in $\left[1_{p}^{1}, 5_{p}^{1}\right]$ is $5_{p}>31_{p}>3_{p}>11_{p}>1_{p}$ and according to the bijection in the proof of Proposition 4.14 this chain correspond to the Dyck path UD. The proof also tells us the critical chains of $\left[1_{p}^{2}, 5_{p}^{2}\right]$ are:

$$
\begin{aligned}
& 55_{p}>531_{p}>53_{p}>331_{p}>33_{p}>311_{p}>31_{p}>111_{p}>11_{p} \\
& 55_{p}>531_{p}>53_{p}>511_{p}>51_{p}>311_{p}>31_{p}>111_{p}>11_{p}
\end{aligned}
$$

which correspond to the Dyck paths UUDD and UDUD, respectively.

Proposition 4.16. For any non-negative integers $x+y=n$ :

$$
\mu\left(1_{p}^{n}, 4^{x} 3_{p}^{y}\right)=(-1)^{x}\left(\begin{array}{l}
n \\
y
\end{array}\right) .
$$

Proof. By an analogous argument to that used in the proof of Proposition 4.14 we can see that a critical chain $C$ can only contain $\left(\begin{array}{l}4 \\ 3\end{array}\right)_{\mathrm{p}},\left(\begin{array}{l}3 \\ 11\end{array}\right)_{\mathrm{p}}$ and $\left(\begin{array}{l}1 \\ 0\end{array}\right)_{\mathrm{p}}$. Moreover, $C$ must have $C\left(\begin{array}{l}4 \\ 3\end{array}\right)_{\mathrm{p}}=x, C\left(\begin{array}{c}3 \\ 11\end{array}\right)_{\mathrm{p}}=n$ and $C\left(\begin{array}{l}1 \\ 0\end{array}\right)_{\mathrm{p}}=n$. Note that $\left(\begin{array}{l}1 \\ 0\end{array}\right)_{\mathrm{p}}$ must be preceded by $\left(\begin{array}{c}3 \\ 11\end{array}\right)_{\mathrm{p}}$, and as $C\left(\begin{array}{c}3 \\ 11\end{array}\right)_{\mathrm{p}}=C\left(\begin{array}{l}1 \\ 0\end{array}\right)_{\mathrm{p}}$ this implies every $\left(\begin{array}{c}3 \\ 11\end{array}\right)_{\mathrm{p}}$ must be followed by a $\left(\begin{array}{l}1 \\ 0\end{array}\right)_{\mathrm{p}}$. So we have $n$ copies of the consecutive pair $\left(\begin{array}{c}3 \\ 11\end{array}\right)_{\mathrm{p}},\left(\begin{array}{l}\mathrm{p} \\ 0\end{array}\right)_{\mathrm{p}}$ and we can choose to insert $\left(\begin{array}{l}4 \\ 3\end{array}\right)$ p before any $\left(\begin{array}{c}3 \\ 11\end{array}\right)$ p so there are $\left(\begin{array}{l}n \\ y\end{array}\right)$ such chains. Table 2 implies that any chain of this form is critical. Finally, the rank equals $3 x+2 y$ so the sign is given by $(-1)^{3 x+2 y}=(-1)^{x}$.

Lemma 4.17. For any $n>0$ we have $\mu\left(1_{p}^{n}, 1_{p}^{n}\right)=1$ and

$$
\mu\left(1_{p}^{n}, 2_{p}^{n}\right)=\left\{\begin{array}{ll}
-1, & \text { if } n=1 \\
0, & \text { if } n>1
\end{array} .\right.
$$


Proof. It is trivial to see that $\mu\left(1_{p}^{n}, 1_{p}^{n}\right)=1$. The interval $\left(1_{p}^{n}, 2_{p}^{n}\right)$ is a chain of the graphs $2^{x} 1^{y}$, with $x+y=n$, which implies the result.

Propositions 4.14 and 4.16 and Lemma 4.17 imply cases $1 \leq x \leq 5$ of Theorem 4.1, so along with Section 4.1 we have finished the proof of Theorem 4.1.

We finish this section with two conjectures. Computational evidence indicates that the value of $\mu\left(1_{p}^{n}, 5^{x} 4_{p}^{y}\right)$ is given by the Schröder numbers, see sequence A088617 in [OEI].

Conjecture 4.18. For any non-negative integers $x+y=n$ :

$$
\mu\left(1_{p}^{n}, 5^{x} 4_{p}^{y}\right)=(-1)^{y} T(n, x),
$$

where $T(n, x)$ are the Schröder numbers.

If we compare Figure 3.1 and Figure 4.2 we notice that the intervals $\left[1_{p}^{1}, H\right]$ and $\left[1_{p}^{1}, 5_{p}^{1}\right]$ are isomorphic. Although computing $\left[1_{p}^{2}, H^{2}\right]$ and $\left[1_{p}^{2}, 5_{p}^{2}\right]$ shows they are not isomorphic for $n=2$. However, computational evidence indicates that $\mu\left(1_{p}^{n}, H^{n}\right)$ is also given by the Catalan number, which gives the following conjecture.

Conjecture 4.19. For any $n>0$ :

$$
\mu\left(1_{p}^{n}, H^{n}\right)=\mu\left(1_{p}^{n}, 5_{p}^{n}\right)=\mathcal{C}_{n}
$$

\section{Further Questions}

In this section we finish with some open problems and conjectures relating to the poset of graphs. A natural question to ask is for what proportion of intervals is the Möbius function non-zero. If we take all intervals $[H, G]$ of simple graphs with $|G| \leq n$ with $n=4,5,6,7$ the proportion of intervals with $\mu(H, G)=0$ are approximately: $16.7 \%, 19.1 \%, 19.0 \%$ and $14.2 \%$, respectively. However this is a small sample and we do not know what happens for larger graphs.

Question 5.1. What proportion of intervals have non-zero Möbius function?

We have shown that the Fibonacci sequence and the Catalan sequence appear in as the Möbius function which poses the following question.

Question 5.2. Can we find other combinatorial sequence which appear as the Möbius function of particular classes of graphs in $\mathcal{G}$ ?

We say the Möbius function is alternating if the sign is given by the rank, that is $\mu(H, G)$ has $\operatorname{sign}(-1)^{|G|-|H|}$. This is not always true in $\mathcal{G}$. For example, let $G$ be the graph in Figure 5.1 , then $\mu\left(\bar{K}_{2}, G\right)=1$ so the sign is not given by $(-1)^{7-2}$.

If the sign of the Möbius function is not determined by the parity of the rank, then the interval is not shellable. We know that an interval is not shellable if it contains a non-trivial disconnected subinterval. 


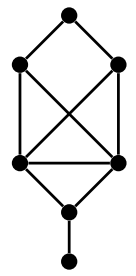

Figure 5.1: A graph $G$ for which $\mu\left(\overline{K_{2}}, G\right)=1$.

Question 5.3. Are there any other obstructions to shellability in $\mathcal{G}$ and $\mathcal{G}^{c}$ and what proportion of intervals are shellable?

For many posets of combinatorial objects it is conjectured that rank functions of all intervals are unimodal, but this is often difficult to prove. All the intervals of $\mathcal{G}$ and $\mathcal{G}^{c}$ that we have computed thus far have been unimodal, although this is still a very small sample size.

Conjecture 5.4. All intervals of $\mathcal{G}$ and $\mathcal{G}^{c}$ are unimodal.

Studying intervals of $\mathcal{G}$ has links to the graph reconstruction problem, which states that every graph $G$ is uniquely determined by the set of graphs obtained by deleting a single vertex from $G$, see [Bon91]. If the reconstruction conjecture is true, it implies the set of coatoms of $\left[K_{1}, G\right]$ are unique for every $G$ with $|G|>2$, where the coatoms are the maximal elements of the interior. But is this true if we change the bottom graph of the interval?

Question 5.5. Is the set of coatoms of $[H, G]$ unique for every pair $H<G$ ?

\section{Acknowledgements}

I would like to express my gratitude to the anonymous referees for their extremely useful comments and corrections which greatly improved the paper.

\section{References}

[BH05] Eric Babson and Patricia Hersh. Discrete Morse functions from lexicographic orders. Transactions of the American Mathematical Society, 357(2):509-534, 2005.

[BJJS11] Alexander Burstein, Vít Jelínek, Eva Jelínková, and Einar Steingrímsson. The Möbius function of separable and decomposable permutations. Journal of Combinatorial Theory. Series A, 118(8):23462364, 2011.

[Bjö80] Anders Björner. Shellable and Cohen-Macaulay partially ordered sets. Transactions of the American Mathematical Society, 260(1):159-183, 1980. 
[Bjö90] Anders Björner. The Möbius function of subword order. Institute for Mathematics and its Applications, 19:118, 1990.

[BM76] John Adrian Bondy and Uppaluri Siva Ramachandra Murty. Graph theory with applications, volume 6. Macmillan London, 1976.

[Bon91] John Adrian Bondy. A graph reconstructor's manual. Surveys in combinatorics, pages 221-252, 1991.

[Die12] Reinhard Diestel. Graph Theory, 4th Edition, volume 173 of Graduate texts in mathematics. Springer, 2012.

[For95] Robin Forman. A discrete morse theory for cell complexes. In in Geometry, Topology 6 Physics for Raoul Bott. Citeseer, 1995.

[Koz08] Dmitry Kozlov. Combinatorial Algebraic Topology. Algorithms and Computation in Mathematics. Springer, 2008.

[KS96] André E. Kézdy and Steve Seif. When is a poset isomorphic to the poset of connected induced subgraphs of a graph? Southwest J. Pure Appl. Math, 1, 1996.

[MS15] Peter R. W. McNamara and Einar Steingrímsson. On the topology of the permutation pattern poset. Journal of Combinatorial Theory, Series A, 134:1-35, 2015.

[OEI] The On-Line Encyclopedia of Integer Sequences. Published electronically at http://oeis.org.

[RS04] Neil Robertson and Paul D Seymour. Graph minors. xx. Wagner's conjecture. Journal of Combinatorial Theory, Series B, 92(2):325$357,2004$.

[Smi16a] Jason P. Smith. Intervals of permutations with a fixed number of descents are shellable. Discrete Mathematics, 339(1):118 - 126, 2016.

[Smi16b] Jason P. Smith. On the Möbius function and topology of general pattern posets. arXiv preprint arXiv:1705.08676, 2016.

[Smi17] Jason P Smith. A formula for the Möbius function of the permutation poset based on a topological decomposition. Advances in Applied Mathematics, 91:98 - 114, 2017.

[ST17] Stephen GZ Smith and István Tomon. The poset on connected graphs is sperner. Journal of Combinatorial Theory, Series A, 150:162-181, 2017.

[SV06] Bruce E. Sagan and Vincent Vatter. The Möbius function of a composition poset. Journal of Algebraic Combinatorics, 24(2):117-136, 2006. 
[Tha06] Bhalchandra D Thatte. Subgraph posets and graph reconstruction. arXiv preprint math/06095\%4, 2006.

[Tho87] Carsten Thomassen. A characterization of locally finite vertextransitive graphs. Journal of Combinatorial Theory, Series B, 43(1):116 - 119, 1987.

[Wac07] Michelle L. Wachs. Poset topology: Tools and applications. In Geometric Combinatorics, volume 13 of IAS/Park City Math. Ser., pages 497-615. Amer. Math. Soc., 2007.

[Wir16] Alexander Wires. Definability in the substructure ordering of simple graphs. Annals of Combinatorics, 20(1):139-176, 2016. 